\title{
Volume holographic hyperspectral imaging
}

\author{
Wenhai Liu, George Barbastathis, and Demetri Psaltis
}

\begin{abstract}
A volume hologram has two degenerate Bragg-phase-matching dimensions and provides the capability of volume holographic imaging. We demonstrate two volume holographic imaging architectures and investigate their imaging resolution, aberration, and sensitivity. The first architecture uses the hologram directly as an objective imaging element where strong aberration is observed and confirmed by simulation. The second architecture uses an imaging lens and a transmission geometry hologram to achieve linear two-dimensional optical sectioning and imaging of a four-dimensional (spatial plus spectral dimensions) object hyperspace. Multiplexed holograms can achieve simultaneously three-dimensional imaging of an object without a scanning mechanism. (C) 2004 Optical Society of America

OCIS codes: $\quad 090.1970,090.4220,090.7330,180.6900,100.6890,170.6280$.
\end{abstract}

\section{Introduction}

Holography has been intensively investigated as a potential information storage technology ${ }^{1-8}$ and for information-processing elements, ${ }^{9}$ such as in neural networks, ${ }^{10-12}$ optical correlators, ${ }^{13-15}$ optical interconnections, ${ }^{16-19}$ and diffracting elements. ${ }^{20-23}$

Optical information storage and optical information processing have two significantly different holographic philosophies. For a holographic memory system a complex optical pattern encoded with information to be stored is recorded inside the hologram medium. The information is retrieved later by reconstructing the optical pattern with its corresponding simple predesigned reference beam. For a holographic information-processing system a predesigned holographic pattern is stored first in the medium and then probed by an incident signal with complex optical fields. The predesigned hologram extracts certain components from the complex input by Bragg phase matching and diffracts them as specific reference wave fronts. Holographic resolution, or Bragg phase selectivity in spatial and spectral di-

W. Liu is with Ondax, Inc., 850 East Duarte Road, Monrovia, California 91016 (e-mail, wliu@ondax.com). G. Barbastathis is with the Department of Mechanical Engineering, Massachusetts Institute of Technology, 3-461C, 77 Massachusetts Avenue, Cambridge, Massachusetts 02139 (e-mail, gbarb@mit.edu). D. Psaltis is with the Department of Electrical Engineering, California Institute of Technology, Pasadena, California 91125 (e-mail, psaltis@sunoptics.caltech.edu).

Received 4 November 2003; revised manuscript received 17 February 2004; accepted 12 March 2004.

0003-6935/04/183581-19\$15.00/0

(C) 2004 Optical Society of America mensions, plays a key role in both applications. It determines the information capacity to be stored in and reconstructed from the holographic memory or the information capacity to be extracted and processed by a hologram from the complex signal input.

Modern imaging technology is widely used in all areas of daily life and scientific practice where image information is projected by an optical system onto a two-dimensional (2-D) image sensor, such as photographic film, CCD's, and complementary metal-oxide semiconductor devices. To extract information from a three-dimensional (3-D) spatial object, an instrument is required to have optical-sectioning capability, i.e., to be able to extract information selectively from 3 -D information and to project it correspondingly onto 2-D sensor. Current imaging optics can only extract the information of a single 2-D section from 3 -D spaces, such as a normal microscope with an imaging lens of small focal depth or information from a single point, such as a confocal microscope with a pinhole spatial filter and a near-field microscope with a point light source. To extract 3-D information, the microscope needs a scanning mechanism, either mechanical scanning, such as the scanning of objective lens focal depth, illumination point source scanning, ${ }^{24,25}$ and laser scanning in a confocal microscope, ${ }^{26,27}$ or optical scanning, such as coherent phase-interference scanning. ${ }^{28,29}$

When the abilities of selective wave-front processing and multiplexing are combined, volume holograms can achieve multidimensional tomographic imaging. ${ }^{30,31}$ In this application, predesigned diffraction patterns are recorded as holograms inside the holographic material. Signals from an object with unknown wave front or information illuminate 
the holograms and are diffracted, filtered, and optically processed by the holographic pattern because of selective Bragg phase matching. By properly designing the holograms and the optical architecture, it is possible to use a hologram as an imaging element to extract and form an image onto an image sensor. The key advantages are as follows:

(1) Volume holograms are selective diffraction elements owing to sensitive Bragg phase matching. For a certain hologram only the Bragg-matched wave front is diffracted strongly and extracted with no other signal component affected.

(2) Multiplexing ability makes it possible to process different wave-front components at the same time without their affecting one another.

In this paper we study theoretically and experimentally two holographic architectures used for 3-D hyperspectral imaging. Their spatial-spectral resolution and image distortion are simulated and compared with experimental measurements. Using multiplexed holograms, we demonstrate real-time 3-D imaging of fluorescent microspheres. To our knowledge this technique is currently the only method that allows simultaneous 3-D spatial imaging and spectroscopic imaging without a scanning mechanism.

\section{Theoretical Principles}

A. Three-Dimensional Imaging and the Confocal Microscope

An optical imaging system projects information about an object to the detector by applying an analog linear transformation on the transverse field intensity distribution through optical elements, as shown in Fig. 1.

The light distribution around the geometrical focal plane was first calculated by Zernike and Nijboer in 1949. ${ }^{32}$ Considering the light distribution near focal point $\mathrm{O}$ of an aberration-free lens with a circular aperture, the light intensity at point $\mathrm{P}$ relative to focus point $\mathrm{O}$ with a depth transition $z$ and transverse distance $r$ is given $\operatorname{as}^{32}$

$$
\begin{aligned}
I(u, v) & =\left(\frac{2}{u}\right)^{2}\left[U_{1}^{2}(u, v)+U_{2}^{2}(u, v)\right] I_{0}, \\
u & =\frac{2 \pi}{\lambda}\left(\frac{a}{f}\right)^{2} z, \\
v & =\frac{2 \pi}{\lambda}\left(\frac{a}{f}\right) r \\
I_{0} & =\left(\frac{\pi a^{2}|A|^{2}}{\lambda f^{2}}\right)^{2}, \\
U_{n}(u, v) & =\sum_{s=0}^{\infty}(-1)^{s}\left(\frac{u}{v}\right)^{n+2 s} J_{n+2 s}(v),
\end{aligned}
$$

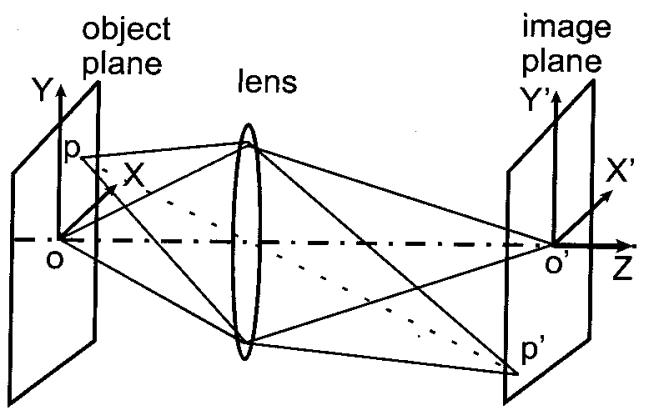

Fig. 1. Planar imaging by an optical lens.

where $a$ is the radius of the circular aperture, $A$ is the light amplitude at the aperture, $f$ is the lens focal length, $U_{n}$ represents Lommel functions, and $J_{n}$ is the Bessel functions.

For the light-intensity distribution in the geometrical focal plane, $z=0$,

$$
I(0, v)=\left[\frac{2 J_{1}(v)}{v}\right]^{2} I_{0},
$$

which is the familiar Airy formula determining the image resolution:

$$
\delta r=0.610 \frac{\lambda}{N A},
$$

$$
N A=\frac{a}{f} .
$$

For the light intensity along the axis, $v=0$,

$$
I(u, 0)=\left[\frac{\sin (u / 4)}{u / 4}\right]^{2} I_{0},
$$

where the first null is

$$
\delta z=\frac{2 \lambda}{(N A)^{2}}
$$

with numerical aperture NA in Eq. (8).

For the traditional concept, where a loss of $20 \%$ in intensity from the center of the image is regarded as permissible,$^{32}$ the focal tolerance $\delta z$ for $I(u, 0) \simeq 0.8 I_{0}$ is given as

$$
\delta z^{\prime} \simeq \frac{0.5 \lambda}{(N A)^{2}} .
$$

The transverse resolution and the focal-depth tolerance determine the spatial resolution of the object in 3-D space, which is called voxel, when the object is illuminated uniformly. Because of the shift invariance in the transverse dimension, a transverse optical section across the focal point is projected onto the image plane as pixels with resolution determined by $\delta r$ in Eq. (7). All the light from other locations in the 3 -D object is out of focus and generates a relatively uniform background on the image plane, which determines the image contrast and signal-to-noise ra- 


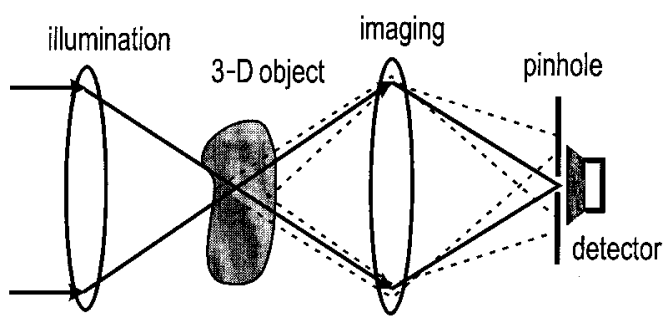

Fig. 2. Confocal microscope architecture.

tio. For full 3-D spatial information, scanning along the axis direction is required.

In the confocal microscope in Fig. 2 the object is illuminated by a focused beam, which gives the same spatial-intensity illumination profile as determined by Eq. (1). When the scattered signal is collected, the intensity distribution function at the image plane is $I(u, v)^{2}$, which leads to a higher transverse and depth resolution for each voxel, at the expense of detecting a single voxel at a time. When a pinhole of the same size is used as the transverse resolution to filter the image point in front of the detector, the stray lights from the different locations except the illuminated focused point are blocked, which achieves a high signal-to-noise ratio for the intensity from the focused object point. Three-dimensional scanning is required for full spatial information.

\section{B. Imaging with Volume Holograms}

Because of Bragg phase matching, a volume hologram can selectively diffract a certain wave pattern while leaving other components in the signal beam untouched. This can serve as a good spatial or spectral filter replacing the imaging pinhole in a traditional confocal microscope. ${ }^{33}$ A confocal microscope with volume holograms as the filter instead of a pinhole has been demonstrated. ${ }^{34}$ In this holographic imaging system a hologram was recorded with a point source and its corresponding coherent reference beam, as shown in Fig. 3. The recording signal beam from a point source $\left(x_{r}, y_{r}, z_{r}, \lambda_{r}\right)$ and its coherent reference plane wave beam along the $\hat{\mathbf{x}}$ axis with a small angle $u \ll 1$ are

$$
\begin{aligned}
E_{s}\left(\mathbf{r} ; \mathbf{r}_{r}, \lambda_{r}\right) & =\exp \left[i \mathbf{k}_{s} \cdot\left(\mathbf{r}-\mathbf{r}_{r}\right)\right], \\
\mathbf{k}_{s}\left(\mathbf{r} ; \mathbf{r}_{r}, \lambda_{r}\right) & =\frac{2 \pi}{\lambda_{r}} \frac{\mathbf{r}-\mathbf{r}_{r}}{\left|\mathbf{r}-\mathbf{r}_{r}\right|}, \\
E_{\mathrm{ref}}\left(\mathbf{r}, \lambda_{r}\right) & =\exp \left(i \mathbf{k}_{\mathrm{ref}} \cdot \mathbf{r}\right), \\
\mathbf{k}_{\mathrm{ref}}\left(\lambda_{r}\right) & =\frac{2 \pi}{\lambda_{r}}\left[-\left(1-\frac{u^{2}}{2}\right)^{2} \hat{\mathbf{x}}+u \hat{\mathbf{z}}\right] .
\end{aligned}
$$

The recorded modulation of the material refractive index inside the material is given to the first order as

$$
\begin{aligned}
\Delta \epsilon(\mathbf{r}) & =\left|E_{\mathrm{ref}}(\mathbf{r})+E_{s}(\mathbf{r})\right|^{2} \\
& \sim E_{s}^{*}(\mathbf{r}) E_{\mathrm{ref}}(\mathbf{r}),
\end{aligned}
$$

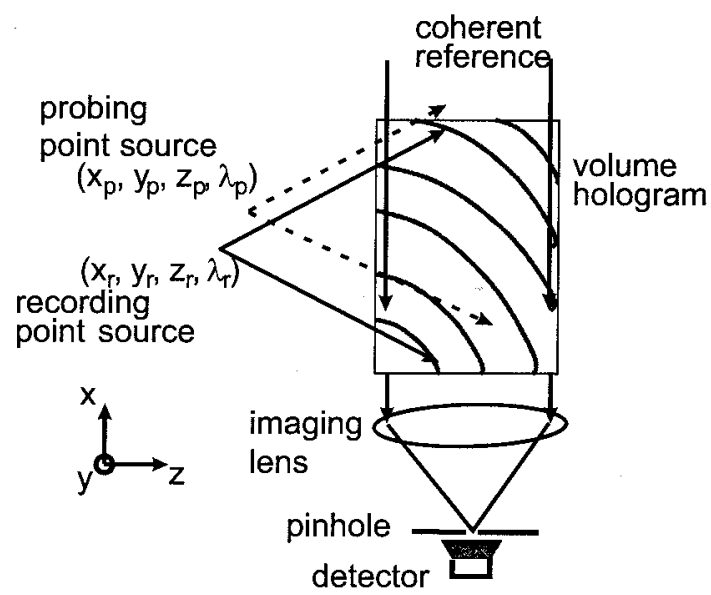

Fig. 3. Recording of a volume holographic spatial filter with a point source and its corresponding coherent plane-wave reference beam in 90-deg geometry. Another point source at a different wavelength is used as a probe.

where the other three terms in the interference pattern are mismatched in our reconstruction.

When a probing point source $\left(x_{p}, y_{p}, z_{p}, \lambda_{p}\right)$ illuminates the volume hologram,

$$
\begin{aligned}
& E_{p}\left(\mathbf{r} ; \mathbf{r}_{p}, \lambda_{p}\right)=\exp \left[i \mathbf{k}_{p} \cdot\left(\mathbf{r}-\mathbf{r}_{p}\right)\right], \\
& \mathbf{k}_{p}\left(\mathbf{r} ; \mathbf{r}_{p}, \lambda_{p}\right)=\frac{2 \pi}{\lambda_{p}} \frac{\mathbf{r}-\mathbf{r}_{p}}{\left|\mathbf{r}-\mathbf{r}_{p}\right|},
\end{aligned}
$$

the diffracted field integral over the holographic grating is given according to the first-order Born approximation as

$E_{d}\left(\mathbf{r}^{\prime}\right)=\iiint_{V} E_{p}(\mathbf{r}) \Delta \epsilon(\mathbf{r}) \exp \left(i 2 \pi \frac{\left|\mathbf{r}-\mathbf{r}^{\prime}\right|}{\lambda_{p}}\right) \mathrm{d}^{3} \mathbf{r}$.

The diffraction is phase matched and significant when the probing point source is identical to the recording source $\left(x_{r}, y_{r}, z_{r}, \lambda_{r}\right)=\left(x_{p}, y_{p}, z_{p}, \lambda_{p}\right)$. Otherwise the diffraction is Bragg mismatched and can be ignored. This phenomenon is called angle, shift, or wavelength selectivity depending on the changes between the recording and probing sources. This sensitive spatial and spectral selectivity is the main motivation for using a volume hologram as a spatial filter in confocal microscopes. In addition to the spatial filtering the hologram diffraction also depends on the wavelength of the recording and the probing beams. Therefore the confocal microscope with a volume hologram actually detects the light intensity from a voxel in 3-D object space within a certain wavelength range $\Delta \lambda$, determined by the volume hologram wavelength selectivity. This 3 -D spatial voxel plus spectral resolution is defined as a texel in four-dimensional (4-D) hyperspace.

There are normally two degenerate directions existing in a single hologram in which signals are diffracted significantly. One is the direction out of the signal-reference plane, along which the wave-vector changes are minimized in the $\mathrm{K}$ sphere. Another is 


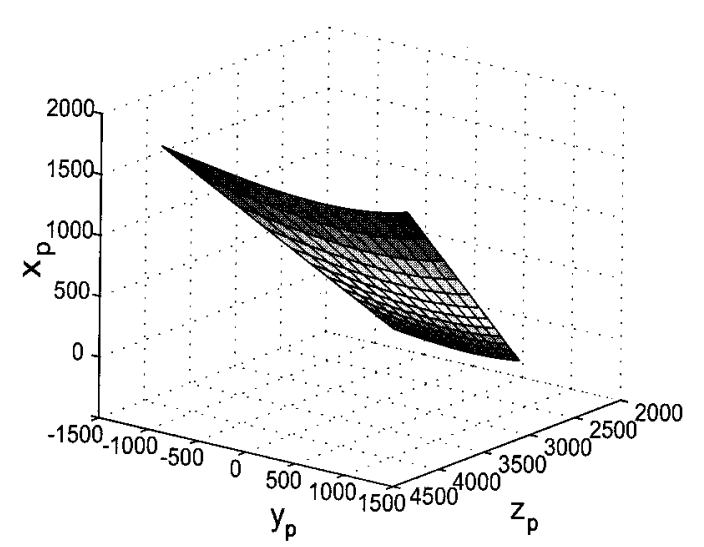

Fig. 4. Numerically calculated degenerate surface (space and color) of the architecture in Fig. 3. The shape in the figure represents the shape of the 2-D slice that the sensor extracts from the 4-D object and projects onto a contiguous area of the detector. Each point on the grid represents one object texel and its corresponding pixel on the detector. ${ }^{30}$

a combination with wavelength and spatial shifting, which forms a new Bragg phase-matching condition for the same holographic grating in a different $K$ sphere. These degeneracies are normally avoided in holographic storage applications because of the cross talk between difference data pages during reconstruction. However, these invariances provide a hologram the capability of optically sectioning a 2-D slice from the 4-D hyperspace of an object (3-D spatial plus spectrum), compared with single-voxel imaging in confocal microscopes.

With the geometry of Fig. 3, numerical studies ${ }^{30}$ have shown that optically sectioning a curved surface in the 3-D object spatial and spectral space in Fig. 4 can be achieved by a single hologram recorded with a point source at a certain wavelength.

By the multiplexing of different holograms recorded with point sources at different spatial locations and wavelengths, multiple sections from the object's 4-D hyperspace can be obtained and projected onto a different portion of a $2-\mathrm{D}$ sensor, leading to an imaging system with the ability to extract information from 4-D space without the need for a scanning mechanism, as shown in Fig. 5.

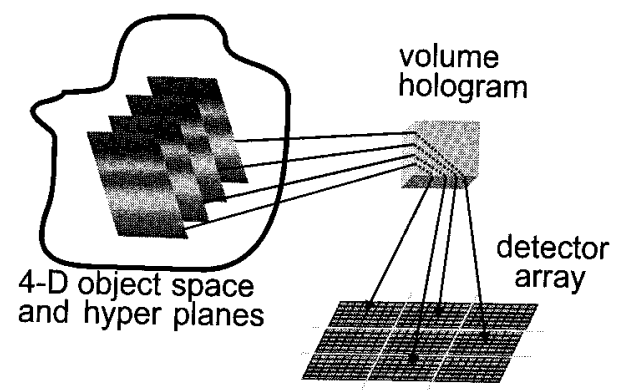

Fig. 5. Multiple-volume holograms optically sectioning different hyperplanes from a 4-D object hyperspace and projecting them onto different 2-D image sensors.

\section{Numerical Mode}

The 3-D spatial diffraction by a holographic pattern with a finite volume is a difficult mathematical problem because of the 3-D dependence. Considering the 90-deg geometry in Fig. 3 as one example, one determines the diffraction field by a probing beam $\left(x_{p}, y_{p}\right.$, $z_{p}, \lambda_{p}$ ) by Eq. (20) by using the Born approximation. There is no simple analytic solution to the $3-\mathrm{D}$ spatial integral for a complex holographic grating inside the material. Barbastathis and Brady ${ }^{30}$ simplified the 3-D spatial integral down to a one-dimensional integral by using paraxial and geometrical approximations. However, the formulas are still mathematically sophisticated, not physically intuitive, and hard to apply to different holographic geometries because of the specific geometry dependency in the formula.

Here we develop a simple numerical model for the 3 -D spatial numerical integral with intuitive physical meaning and little computing time and memory requirement. It applies to any geometric structure and can simulate the diffracted spatial distribution pattern for weak holographic recording and reconstruction close to the Bragg-phase-matching condition.

As shown in Fig. 6, we are interested in calculating the intensity pattern for the $\mathbf{y}^{\prime \prime}-\mathbf{z}^{\prime \prime}$ Fourier plane image by a lens of focal length $f$, which is determined by the Fourier transformation of Eq. (20):

$$
\begin{aligned}
E_{F}\left(x^{\prime}-f, y^{\prime \prime}, z^{\prime \prime}\right)= & \iint_{-\infty}^{\infty} E_{d}\left(x^{\prime} ; y^{\prime}, z^{\prime}\right) \\
& \times \exp \left(-i 2 \pi \frac{y^{\prime} y^{\prime \prime}+z^{\prime} z^{\prime \prime}}{\lambda_{p} f}\right) \mathrm{d} y^{\prime} \mathrm{d} z^{\prime}, \\
= & \iiint_{V} d^{3} \mathbf{r} E_{p}\left(\mathbf{r}, \lambda_{p}\right) E_{s}^{*}\left(\mathbf{r} ; \mathbf{r}_{r}, \lambda_{r}\right) \\
& \times E_{\mathrm{ref}}\left(\mathbf{r}, \lambda_{r}\right) \\
& \times E_{d}{ }^{*}\left(\mathbf{r} ; \mathbf{r}^{\prime \prime}, \lambda_{p}\right),
\end{aligned}
$$

where the diffracted wave front $E_{d}\left(\mathbf{r} ; \mathbf{r}^{\prime \prime}, \lambda_{p}\right)$ is defined as

$$
E_{d}\left(\mathbf{r} ; \mathbf{r}^{\prime \prime}, \lambda_{p}\right)=\exp \left[i \mathbf{k}_{d}\left(\mathbf{r}^{\prime \prime}, \lambda_{p}\right) \cdot \mathbf{r}\right]
$$

i.e., a plane wave propagating along the $-\hat{\mathbf{x}}$ direction with transverse spatial frequency $\left(y^{\prime \prime} / f\right) k \hat{\mathbf{y}}+\left(z^{\prime \prime}\right)$ f) $k \hat{\mathbf{z}}$ at wavelength $\lambda_{p}$ :

$$
\mathbf{k}_{d}\left(\mathbf{r}^{\prime \prime}, \lambda_{p}\right)=\frac{2 \pi}{\lambda_{p}}\left[-\left(1-\frac{y^{\prime \prime 2}}{2 f^{2}}-\frac{z^{\prime \prime 2}}{2 f^{2}}\right) \hat{\mathbf{x}}+\frac{y^{\prime \prime}}{f} \hat{\mathbf{y}}+\frac{z^{\prime \prime}}{f} \hat{\mathbf{z}}\right] .
$$




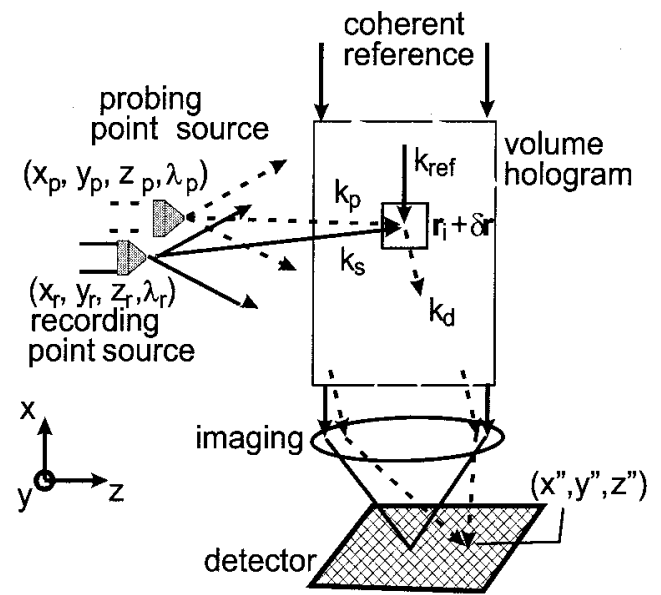

Fig. 6. Three-dimensional integral of the small cubic volume of the holographic grating. The small cubic volume $\Delta V=\Delta x \times \Delta y \times$ $\Delta z$ is centered at $\mathbf{r}_{i}$. The full integral over the volume $V$ is $\iiint_{\mathrm{V}} \mathrm{d}^{3} \mathbf{r}=\Sigma_{i} \iiint_{\Delta V_{i}} \mathrm{~d}^{3} \boldsymbol{\delta} \mathbf{r}$.

This leads the diffracted image pattern to the typical form of four-wave mixing, or a holographic Bragg-phase-matching integral:

$$
\begin{aligned}
E_{F}\left(x^{\prime}-f, y^{\prime \prime}, z^{\prime \prime}\right)= & \iiint_{V} d^{3} \mathbf{r} \exp \left[i \mathbf{k}_{p} \cdot\left(\mathbf{r}-\mathbf{r}_{p}\right)\right. \\
& \left.-i \mathbf{k}_{s} \cdot\left(\mathbf{r}-\mathbf{r}_{r}\right)\right] \exp \left[i \left(\mathbf{k}_{\mathrm{ref}}\right.\right. \\
& \left.\left.-\mathbf{k}_{d}\right) \cdot \mathbf{r}\right]
\end{aligned}
$$

The integral is maximized in the Bragg-phasematching condition when the phase factor is

$$
\begin{aligned}
\phi= & \mathbf{k}_{p}\left(\mathbf{r} ; \mathbf{r}_{p}, \lambda_{p}\right) \cdot\left(\mathbf{r}-\mathbf{r}_{p}\right)-\mathbf{k}_{s}\left(\mathbf{r} ; \mathbf{r}_{r}, \lambda_{r}\right) \cdot\left(\mathbf{r}-\mathbf{r}_{r}\right) \\
& +\left(\mathbf{k}_{\mathrm{ref}}-\mathbf{k}_{d}\right) \cdot \mathbf{r}, \\
= & 0
\end{aligned}
$$

For simple geometries, where the four-wave vectors $\mathbf{k}_{p}, \mathbf{k}_{s}, \mathbf{k}_{\text {ref }}$, and $\mathbf{k}_{d}$ are simple functions of $\mathbf{r}$, the diffracted field can be integrated analytically from Eq. (25). For more general conditions, such as the spherical waves in Fig. 6, no simple analytic form can be achieved because of the complex functions for $\mathbf{k}_{p}$, $\mathbf{k}_{s}$ in Eqs. (13) and (19). Only a numerical solution is possible.

To simplify the numerical integral, we consider the integral in Eq. (25) over a small cubic volume $\Delta V_{i}$ centered around $\mathbf{r}_{i}$ only, as shown in Fig. 6 . For point sources at $\mathbf{r}_{r}, \mathbf{r}_{p}$ the integral area $\Delta V_{i}$ is within the far field and the wave front is approximately a plane wave ${ }^{35,36}$ with constant wave vectors in Eqs. (13) and (19) within $\Delta V_{i}$. This reduces the
3-D integral of the diffracted field in Eq. (25) to a finite summation over a finite volume:

$$
\begin{aligned}
E_{F}\left(x^{\prime}-f, y^{\prime \prime}, z^{\prime \prime}\right)= & \sum_{i} \exp \left[i \phi_{i}\left(\mathbf{r}_{i}\right)\right] \iiint_{\Delta V_{i}} \\
& \times d^{3} \boldsymbol{\delta} \mathbf{r} \exp \left(i \Delta \mathbf{K}_{i} \cdot \boldsymbol{\delta} \mathbf{r}\right), \\
= & \sum_{i} \exp \left[i \phi_{i}\left(\mathbf{r}_{i}\right)\right] \Delta V \\
& \times \operatorname{sinc}\left(\frac{\Delta K_{i x} \Delta X}{2 \pi}\right) \operatorname{sinc}\left(\frac{\Delta K_{i y} \Delta Y}{2 \pi}\right) \\
& \times \operatorname{sinc}\left(\frac{\Delta K_{i z} \Delta Z}{2 \pi}\right),
\end{aligned}
$$

where the small cubic volume $\Delta V=\Delta X \times \Delta Y \times \Delta Z$.

Equation (29) provides the basis for our numerical model, which can be used at all different simple geometries that we consider for holographic imaging applications, as long as the far field and the Born approximations are valid. The validity of the model is checked numerically by verifying well-known simple holographic geometries, such as the angle and wavelength selectivity of plane waves in transmission, reflection, and 90-deg geometry. ${ }^{37}$ Also the simulation on holographic imaging is compared and proved to be consistent with experimental measurements in Sections 3-5, where we investigate in detail two holographic imaging structures with and without an objective lens.

\section{Imaging Properties of a 90-Deg Geometry Volume Hologram}

Previous theoretical studies of the 90-deg geometry predicted an optical cross section in the 3-D spatial plus a spectral object hyperspace, ${ }^{30}$ as shown in Figs. 3 and 4. The optical-sectioning resolution, or the minimum distinguishable texel dimension by a volume hologram, is determined by the spatial angle, shift, and wavelength selectivities. They have been well understood through all the studies of holographic storage applications with various multiplexing mechanisms. ${ }^{16,38-47}$ In all these studies on storage, researchers considered only the intensity diffraction efficiency, neglecting the details of the diffracted patterns around the Bragg-mismatching condition. The diffraction patterns are not important to the storage data, which are intended only to be read out at the Bragg-phase-matching condition. However, for the imaging application with a complex signal incident the diffraction pattern around the Bragg-mismatching condition leads to background noise from the mismatching light source next to the Bragg-phase-matching light source. The background determines the imaging contrast and the signal-tonoise ratio.

For data-storage applications the diffraction invariance along the degenerate directions in the optical-sectioning hyperplane is generally not used because of the nonperfect phase-matching condition in degenerate directions. The nonperfect phase 
matching causes the signal-bearing information to be distorted. For some special applications, these phenomena have been investigated and demonstrated, such as the spatial degeneracy in hologram control and optical correlators, ${ }^{13,16}$ or the wavelength degeneracy in two-wavelength nondestructive hologram reconstruction, ${ }^{48-52}$ where special care and design are necessary to correct and control the quality of the reconstructed signal beams.

By making use of the degenerate directions for imaging applications, we expect the hologram diffraction to generate an analog linear transformation from the optical section to the 2-D image sensor. The imaging transformation and aberration along the degenerate direction are more critical than having a strong diffraction efficiency in the optical-section plane. Here we investigate the spatial-spectral selectivity of the 90-deg holographic imaging system in Fig. 6 and study and demonstrate the imaging performance in 90-deg geometry with a spherical wave used as a signal for experiments and numerical simulation.

\section{A. Selectivity by Simulation and Comparison with Experiments}

In the ideal case of Fig. 6, when the probing point source is shifted in either the $\hat{\mathbf{x}}$ or $\hat{\mathbf{z}}$ direction at the same wavelength, there is no diffraction because of Bragg mismatching. When shifting occurs in the $\hat{\mathbf{y}}$ direction at the same wavelength, the diffraction intensity is approximately as strong as the Braggphase-matching condition because of the degeneracies.

The selectivities in the $\hat{\mathbf{x}}, \hat{\mathbf{z}}$ directions and wavelength determine the final resolution of the holographic imaging. From Eq. (29) of the numerical model we simulate the diffraction efficiencies as the probing condition changes and develop a simple approximate analytic model to calibrate the selectivities quantitatively.

\section{Shift Selectivity in the $\hat{\mathbf{x}}$ Direction}

The $\hat{\mathbf{x}}$ direction is the well-studied shift multiplexing direction, ${ }^{13,37,44,46,53}$ where the paraxial approximation or the phase integral along the path gives the approximate theoretical analytic diffraction efficiency as

$$
E_{d} \simeq \int_{-N A / n}^{N A / n} \mathrm{~d} \alpha \exp \left[i \frac{2 \pi n \Delta x}{\lambda} \frac{\alpha}{\left(1+\alpha^{2}\right)^{1 / 2}}\right] .
$$

When the recording-probing beam numerical aperture $N A / n$ is small, using the first-order approximation $\alpha /\left(1+\alpha^{2}\right)^{1 / 2} \approx \alpha$, we simplify Eq. (30) to

$$
\left|E_{d}\right| \simeq \operatorname{sinc}\left(\frac{2 N A \Delta x}{\lambda_{r}}\right)
$$

which gives a sinc function with the first null at

$$
\Delta x=\frac{\lambda_{r}}{2 N A} .
$$

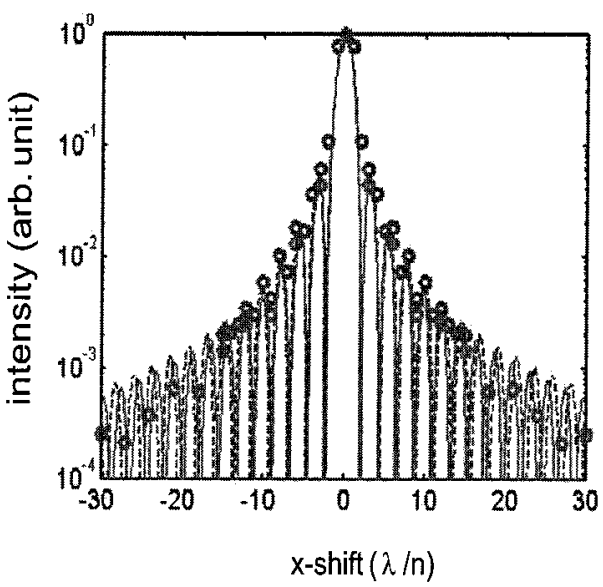

Fig. 7. Numerical simulation and theoretical calculation of the shift selectivity of 90-deg geometry. The simulation parameters are assumed: $\quad N A=0.5, n=2.2: \quad \bigcirc$, numerical simulation; solid curve, theoretical integral of the selectivity curve in Eq. (30); dashed curve, approximation as a sinc function in Eq. (31).

In Fig. 7 the numerical simulation of the diffraction efficiency across the detector is calculated as a function of the horizontal translation $\delta x$ and verifies the approximate theoretical calculation in Eq. (31).

\section{Depth Selectivity along the $\hat{\mathbf{z}}$ Direction}

Using the same phase integral model as for the shift selectivity in the $\hat{\mathbf{x}}$ direction, we can derive and verify with numerical simulation an approximate analytic expression of the depth selectivity. The phase changes along the Bragg-phase-matching direction for depth shifting $\Delta z \ll z_{0}$ and the integral are

$$
\begin{aligned}
\phi\left(\mathbf{r}_{i}, \Delta z\right) & =\frac{2 \pi}{\lambda_{n}} \frac{\Delta z z_{0}}{\left(x^{2}+z_{0}^{2}\right)^{1 / 2}}, \\
E_{d} & \simeq \int_{-N A / n}^{N A / n} \mathrm{~d} \alpha \exp \left[i \frac{2 \pi \Delta z}{\lambda_{n}} \frac{1}{\left(1+\alpha^{2}\right)^{1 / 2}}\right] .
\end{aligned}
$$

The first-order approximation of the integral along the phase-matching direction, $1 /\left(1+\alpha^{2}\right)^{1 / 2} \simeq 1-$ $\alpha^{2} / 2$, leads to the depth selectivity of the diffraction efficiency as Fresnel integrals $C(s), S(s)$ :

$$
\begin{gathered}
\left|E_{d}\right| \simeq \frac{\left[C^{2}(s)+S^{2}(s)\right]^{1 / 2}}{s}, \\
s=N A\left(\frac{2 \Delta z}{n \lambda_{0}}\right)^{1 / 2}, \\
C(s)=\int_{0}^{s} \cos \left(\frac{\pi t^{2}}{2}\right) \mathrm{d} t,
\end{gathered}
$$

$$
S(s)=\int_{0}^{s} \sin \left(\frac{\pi t^{2}}{2}\right) \mathrm{d} t .
$$




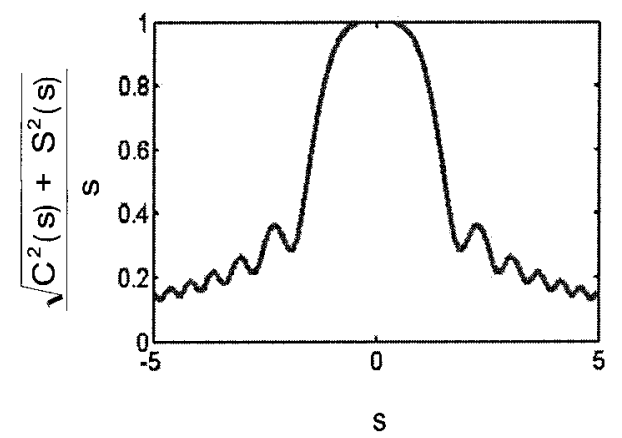

Fig. 8. Function of Fresnel integrals, where the first minimum is at $s=1.91$.

The first minimum of the function $\left[C^{2}(s)+\right.$ $\left.S^{2}(s)\right]^{1 / 2} / s$ occurs at $s=1.91$, as shown in Fig. 8 . This gives for the depth selectivity $\Delta z$,

$$
\Delta z=1.82 \frac{n \lambda_{0}}{N A^{2}}
$$

Figure 9 shows both integrals of depth selectivity in Eq. (34) and the Fresnel integral in Eq. (35). For numerical aperture $N A=0.5, n=2.2$ in the simulation, the Fresnel integral is a good approximation. Both integrals fit well with the numerical simulation results, although the integral on the phase path considers the Bragg-matching direction only while the numerical simulation includes all different diffracted components.

Figure 10 shows experimental measurements on the shift and depth selectivity, which is consistent with the phase integral approximation in Eqs. (31) and (35).

\section{Wavelength Selectivity}

Here we study wavelength selectivity without a suitable tunable laser for the experiments. Figure 11 shows that the selectivity function as the wavelength of the probing beam is different from the original

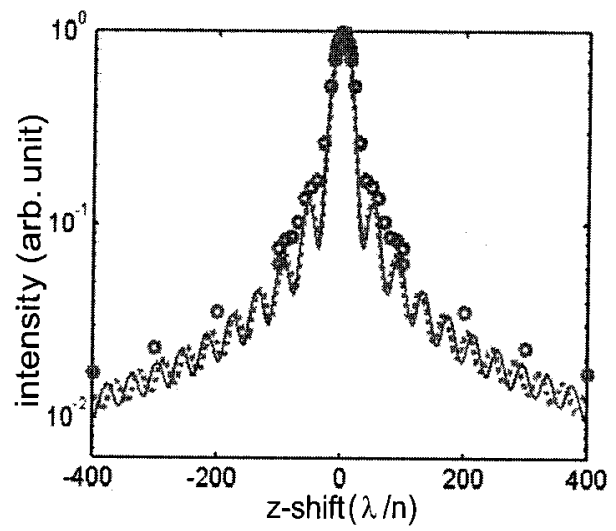

Fig. 9. Numerical simulation and theoretical calculation of depth selectivity of 90-deg geometry. Simulation parameters: $N A=$ $0.5, n=2.2$ : $\bigcirc$, numerical simulation; solid curve, theoretical phase integral of the selectivity curve in Eq. (34); dotted curve, approximation as a Fresnel function in Eq. (35).
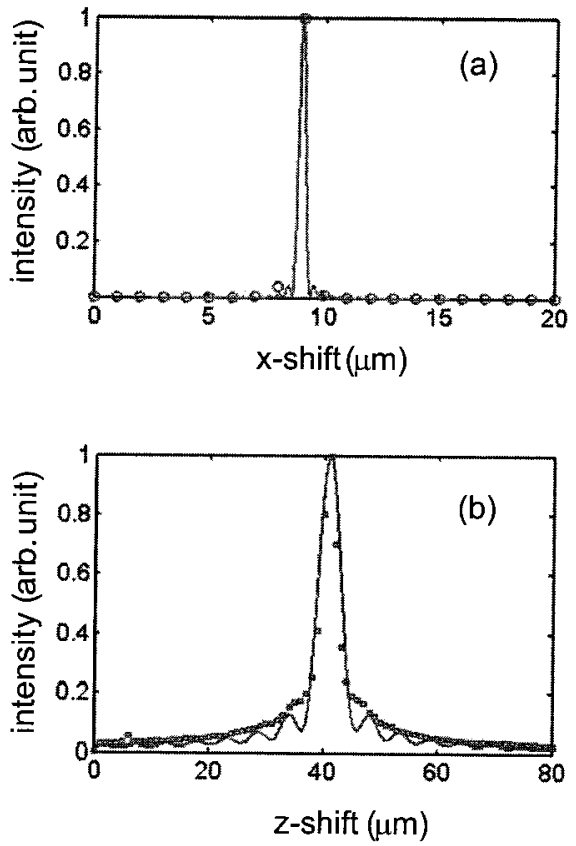

Fig. 10. Experimental measurements of (a) shift selectivity and (b) depth selectivity: $\bigcirc$, experimental measurements; solid curve, their theoretical phase-integral approximation.

recording beam. Also, we can calculate the wavelength selectivity from the approximated phase integral as

$$
\left|E_{d}\right| \simeq \operatorname{sinc}\left(\frac{2 N A z_{0} \Delta \lambda}{\lambda_{0}^{2}}\right)
$$

where the first null for the wavelength selectivity is

$$
\frac{\Delta \lambda}{\lambda_{0}}=\frac{\lambda_{0}}{2 N A z_{0}},
$$

which also depends on depth $z_{0}$.

Considering the thickness of the crystal along the

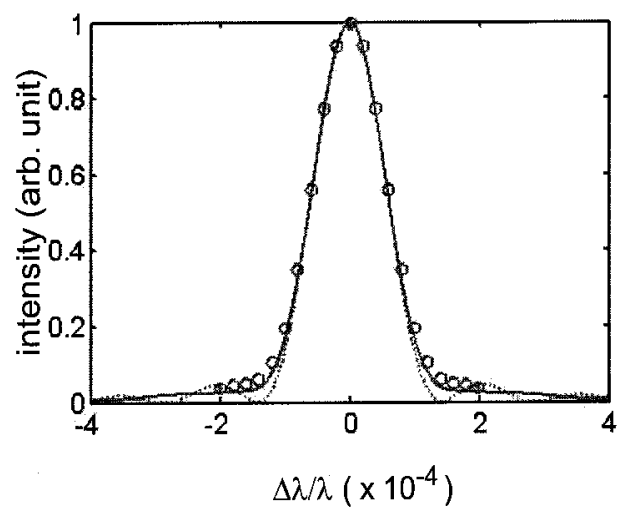

Fig. 11. Numerical simulation and theoretical calculation of the wavelength selectivity of 90-deg geometry. Simulation parameters: $N A=0.5, n=2.2: \quad \bigcirc$, numerical simulation; solid curve, theoretical phase integral of the selectivity curve in Eq. (42); dotted curve, approximation as a sinc function in Eq. (40). 
depth of the signal beam, a more accurate wavelength selectivity is obtained as the average of the sinc function for various depths:

$$
\left|E_{d}\right|^{2} \simeq \frac{1}{z_{2}-z_{1}} \int_{z_{1}}^{z_{2}} \mathrm{~d} z\left|\operatorname{sinc}\left(\frac{2 N A z \Delta \lambda}{\lambda_{0}{ }^{2}}\right)\right|^{2} .
$$

Figure 11 shows both the single sinc function with the depth at the middle of the crystal and the averaged sinc functions. The latter fits better with the numerical simulation results, while the wavelength selectivity in Eq. (41) still gives a reasonable estimation of the wavelength selectivity.

\section{B. Diffracted Image Pattern, Imaging Quality, and Background Noise}

However, the diffracted pattern is not just along the Bragg-phase-matching direction as the phase integral approximation. Instead the diffraction generates a 2-D intensity distribution pattern on the Fourier image plane in Fig. 6. The image pattern of the shift, depth, and wavelength selectivity generates the background of the image, and the patterns along the degenerate direction determine the imaging quality. Numerical simulation of the image patterns helps us understand and design a system for better imaging quality and less background noise.

From Eq. (29) we can simulate the 2-D diffracted patterns of the selectivity conditions in approximations (31), (35), and (42) on the image plane of Fig. 6, as shown in Fig. 12. The image patterns are visually enhanced while their overall intensity follows the selectivity curves in Figs. 7, 9, and 11. The diffraction intensity decreases dramatically when the probe beam is away from the Bragg phase-matching condition, while the pattern intensity distribution changes and spreads out of the original reference focal point, which contributes to the background.

Integrating the 2-D pattern of all the different probing conditions, we can calculate the contrast ratio and resolution of the shift, depth, and wavelength. Consider the case in which the hologram is used as a pinhole for enhancing depth selectivity ${ }^{36}$ and assume that there is a series of object point sources aligned along the $\hat{\mathbf{z}}$ direction with a distance $(10 \lambda) / n\left(n=2.2\right.$ for $\left.\mathrm{LiNbO}_{3}\right)$ between one another. These points are placed outside the depth selectivity $\Delta z$ of Eq. (39), as shown in Fig. 13(a). The central point source is Bragg matched and projects a sharp image onto the $\mathbf{y}^{\prime}-\mathbf{z}^{\prime}$ image plane in Fig. 13(b), while all other point sources generate a small background around the central pixel in Fig. 13(c). A similar calculation can be done in all other spatial-spectral dimensions.

For the imaging application the degenerate direction is expected to deliver a linear transformation from the object space to the image space. Figure 14 shows the simulated image patterns as a probing point source is shifted along the degenerate $\hat{\mathbf{y}}$ direction. The diffraction intensity remains con-

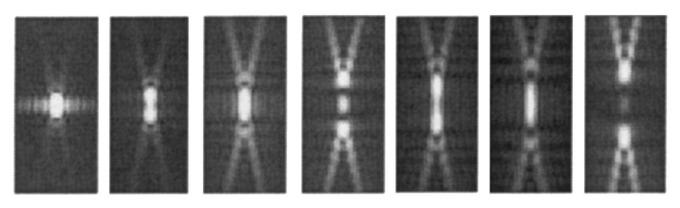

(a)

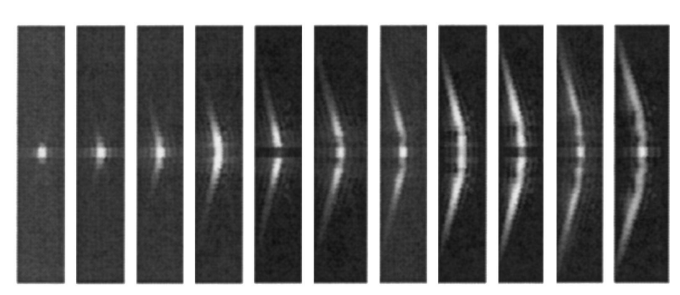

(b)
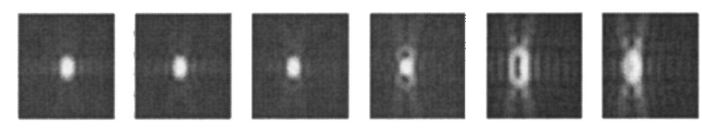

(c)

Fig. 12. Numerical simulation of the image pattern as the probe beam is shifted away from the Bragg-phase-matching condition along the selective directions. Simulation parameters: $N A=$ $0.5, n=2.2$. (a) The probe beam is shifted along the $\hat{\mathbf{x}}$ direction by $(3 \lambda) / n$ for each step, causing weaker and distorted image patterns. (b) The probe beam is shifted along the depth $\hat{\mathbf{z}}$ direction by $(10 \lambda) / n$ for each step. The intensity of the image decay is slower than $\hat{\mathbf{x}}$ shifting, and the intensity deviates dramatically along the $\hat{\mathbf{y}}$ direction. (c) The simulated image pattern as the wavelength of the probing beam shifts from the original recording wavelength by $(\Delta \lambda) / \lambda=4 \times 10^{-5}$ for each pattern.

stant along the degenerate direction, and the image pattern moves linearly as the objective point shifts linearly along $\hat{\mathbf{y}}$. However, the intensitydistribution pattern is severely distorted, as shown in Fig. 14(b), where a linearly aligned point source in an object space along the $\hat{\mathbf{y}}$ direction generates only a decaying butterfly pattern on the image plane.

This aberration indicates that diffraction from the hologram is not necessarily degenerate on the pattern distribution. For a successful imaging system, either a special holographic grating or geometry is necessary to limit the aberration, or a possible digital recovery algorithm is needed to rebuild the information from the aberration.

Figure 15 shows the experimentally measured Fourier plane image patterns when the point source is shifted in either the $\hat{\mathbf{x}}, \hat{\mathbf{y}}$, or $\hat{\mathbf{z}}$ direction at the recording wavelength, which are consistent with the simulations in Figs. 12 and 14.

\section{Imaging Properties of a Transmission Geometry Volume Hologram}

As discussed in Section 3, to obtain a 2-D optical section by using a single hologram, a linear transformation of diffracted image patterns from the object 


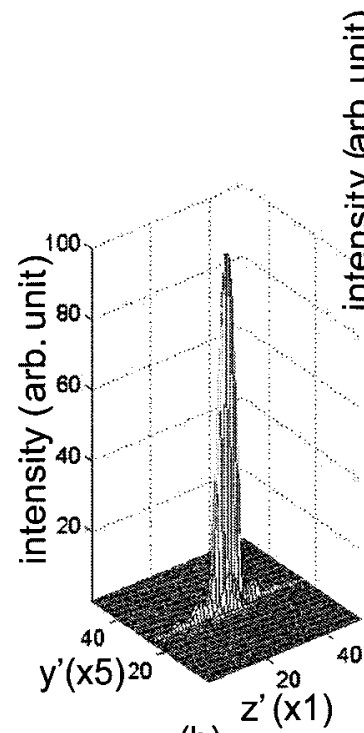

(b)

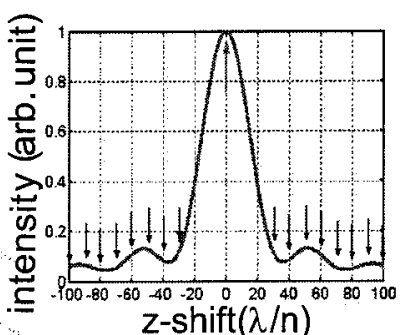

(a)

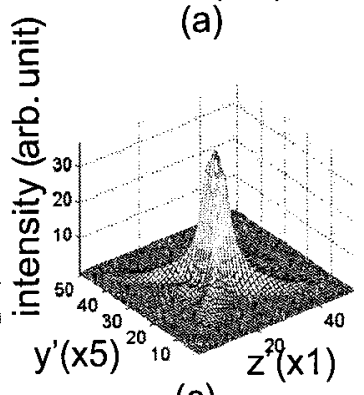

(c)
Fig. 13. Numerical simulation of a series of point sources aligned in the $\hat{\mathbf{z}}$ direction and the image pattern and background. Simulation parameters: $N A=0.5, n=2.2$. (a) Depth-selectivity curves and the location of objective point sources; (b) image intensity pattern for the central Bragg-matched object point source; (c) background pattern from the mismatched object points along the $\hat{\mathbf{z}}$ direction.

space along the degenerate directions is crucial. The spherical wave in 90-deg geometry contains 2-D spatial components, which lead to a sophisticated de-

(a)

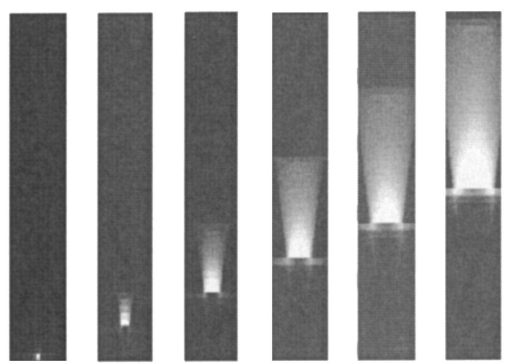

(b)
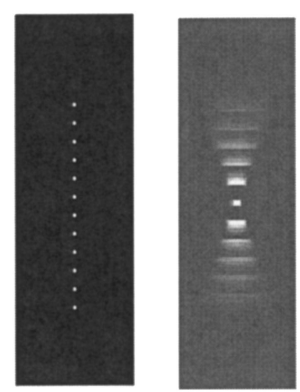

Fig. 14. Numerical simulation of a point source shifting along the degenerate $\hat{\mathbf{y}}$ direction. Simulation parameters: $N A=0.5, n=$ 2.2. (a) Image patterns as a point source shifts along the $\hat{\mathbf{y}}$ direction by $(20 \lambda) / n$ between each step. (b) Image plane responses for a set of point sources linearly aligned on the $\hat{\mathbf{y}}$ direction. The large aberration leads to a decaying butterfly intensity-distributed patterns.

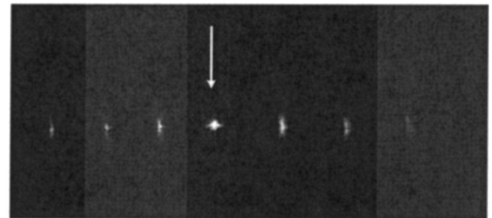

(a)

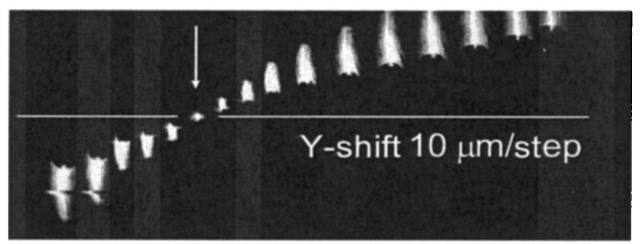

(b)

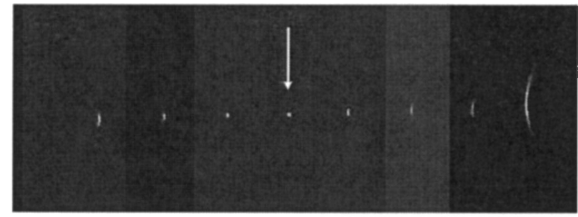

(c)

Fig. 15. Experimental holographic diffraction image patterns in 90-deg geometry with a spherical wave as the signal: (a) image patterns as the signal point source shifts along the $\hat{\mathbf{x}}$ direction, 2 $\mu \mathrm{m}$ for each step; (b) image patterns as the signal point source shifts along the degenerate $\hat{\mathbf{y}}$ direction, $10 \mu \mathrm{m}$ for each step; (c) image patterns as the signal point source shifts along the depth $\hat{\mathbf{z}}$ direction, $6 \mu \mathrm{m}$ for each step. Owing to the selectivity in the $\hat{\mathbf{x}}, \hat{\mathbf{z}}$ directions, the images in (a) and (c) are enhanced for visibility. The arrows point to the Bragg-phase-matching position with the signal at the recording position.

generate diffraction pattern because of the different selectivity effects of various spatial components. This geometry acts better as a spatial filter for diffraction of a single spatial point in a confocal microscope application. ${ }^{34}$ On the other hand, plane waves in a holographic transmission geometry have simple characteristics for degenerate diffraction out of the signal-reference plane. In this section we study the imaging characteristics of the transmission geometry shown in Fig. 16 by the numerical simulation model and demonstrate the 3-D imaging in the experiments.

A collimated signal beam from the point source $\left(x_{r}\right.$, $\left.y_{r}, z_{r}, \lambda_{r}\right)$ and its coherent reference beam generate a simple holographic pattern in the transmission geometry in the photorefractive material with thickness $D$. When the probing beam collimated from a point source $\left(x_{p}, y_{p}, z_{p}, \lambda_{p}\right)$ illuminates the hologram, the diffracted reference is detected at the Fourier plane sensor as a 2-D pattern. In principle, the holographic imaging structure with a collimating lens is the same as the one using spherical waves discussed in Section 3. However, the collimating lens decreases dramatically the spherical curvature of the probe beam near the focus. This leads to a uniform single spatial grating inside the hologram and a linear diffraction transformation along the degenerate 


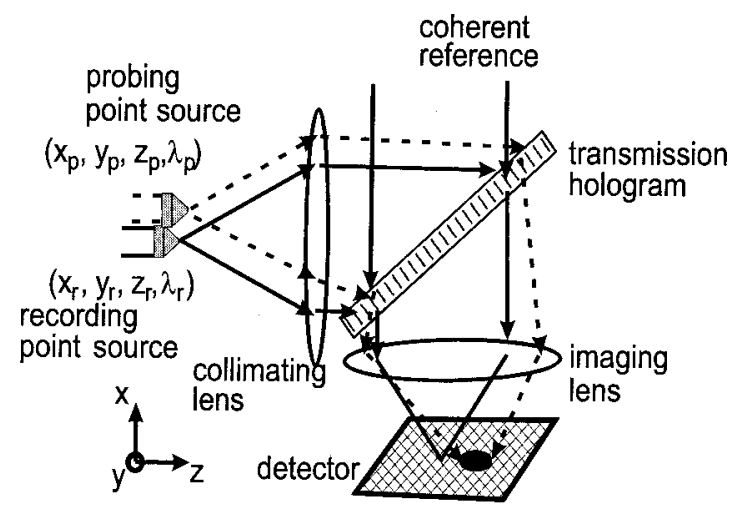

Fig. 16. Transmission geometry hologram recording with point source $\left(x_{r}, y_{r}, z_{r}, \lambda_{r}\right)$ and probing with another point source $\left(x_{p}, y_{p}\right.$, $\left.z_{p}, \lambda_{p}\right)$.

direction, at the cost of an additional collimating lens. This lens also determines the imaging resolution and aberration. We assume that both the collimating and imaging lenses have ideal aberration-free imaging properties in our studies.

\section{A. Selectivity and Diffracted Patterns by Simulation and Experiments}

\section{Shift Selectivity in $\hat{\mathbf{x}}$ :}

Assume that the recording point source is located on the focal point of the collimating lens with focal length $f_{c}$, the recording signal beam coming into the crystal is a plane wave along $\hat{\mathbf{z}}$, where the collimating lens axis is at $x=y=0$. When the probing point shifts by $x_{p}$ in the $\hat{\mathbf{x}}$ direction, the collimated signal beam is tilted by an angle $\left(x_{p} / f_{c}\right)$, where $x_{p} \ll f_{c}$. This leads to the angle selective properties in transmission geometry, which are well known, as shown in the wave-vector $k$ space (Fig. 17).

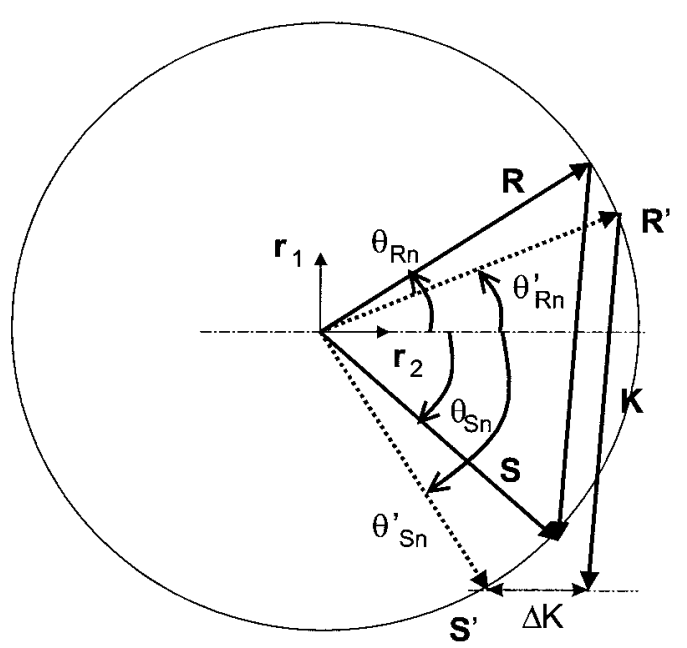

Fig. 17. Angle selectivity of a general transmission geometry hologram by two plane waves $\mathbf{R}, \mathbf{S}$ and readout with a tilted reference $\mathbf{R}^{\prime}$. The hologram is assumed to have an infinite transverse dimension along $\hat{\mathbf{r}}_{1}$ and a thickness $D$ along $\hat{\mathbf{r}}_{2}$. The incident angles for the reference and signal inside the material are $\theta_{R n}, \theta_{S n}$.

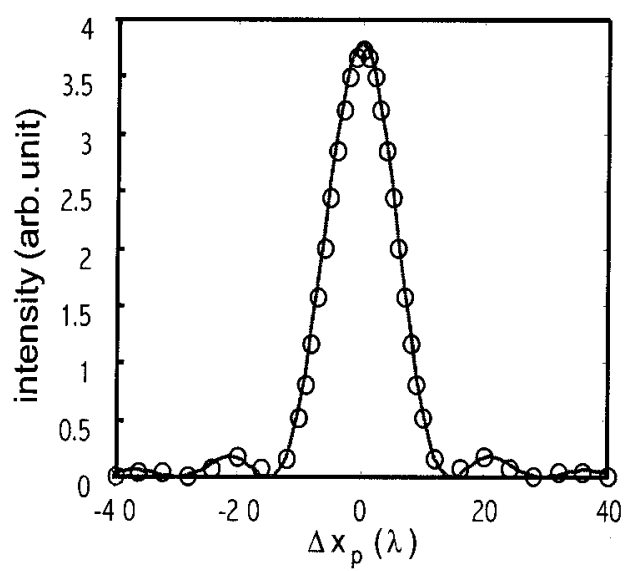

Fig. 18. Simulated angular selectivity of a general transmission geometry hologram in Fig. 16. Simulation condition: $f_{c}=f_{i}=$ $10^{4} \lambda, D=1400 \lambda, N A=0.5, n=2.2, \theta_{R}=\theta_{S}=\pi / 4: \quad \bigcirc$, simulation results of the intensity integral over the 2 -D image pattern on the image plane; solid curve, theoretical $\operatorname{sinc}^{2}$ function in Eq. (49).

Reading out with a reference beam at angle $\theta_{R n}{ }^{\prime}$, we determined the diffracted beam at $\theta_{S n}{ }^{\prime}$ with Bragg-mismatching vector $\Delta K \hat{\mathbf{r}}_{2}$ :

$$
\begin{aligned}
\Delta \theta_{R n} & =\theta_{R n}{ }^{\prime}-\theta_{R n} \ll 1, \\
\Delta \theta_{S n} & =\theta_{S n}{ }^{\prime}-\theta_{S n}, \\
& =-\frac{\cos \theta_{R n}}{\cos \theta_{S n}} \Delta \theta_{R n}, \\
\Delta K & =k_{n}\left(\tan \theta_{S n}+\tan \theta_{R n}\right) \cos \theta_{R n} \Delta \theta_{R n} .
\end{aligned}
$$

This leads to a linear transformation from the object plane point source $\delta\left(\mathbf{r}_{p}-\mathbf{r}_{r}-\Delta x_{p} \hat{\mathbf{x}}, \lambda_{p}=\lambda_{r}\right)$ to a corresponding point image on the image plane $\delta\left(\mathbf{r}_{d}{ }^{\prime}\right.$ $\left.-\mathbf{r}_{r}{ }^{\prime}-\Delta z_{d}{ }^{\prime} \hat{\mathbf{z}}^{\prime}\right)$ of efficiency $\eta$ by the geometry relations in Fig. 16:

$$
\begin{aligned}
\frac{\Delta x_{p}}{f_{c}} \cos \theta_{S} & =-\frac{\Delta z_{d}{ }^{\prime}}{f_{i}} \cos \theta_{R}, \\
\eta & =\operatorname{sinc}^{2}\left(\frac{\Delta K D}{2 \pi}\right), \\
& =\operatorname{sinc}^{2}\left(\frac{\Delta x_{p}}{f_{c} \Delta \alpha}\right), \\
\Delta \alpha & =\frac{\lambda}{D} \frac{1}{\cos \theta_{S}\left(\tan \theta_{S n}+\tan \theta_{R n}\right)},
\end{aligned}
$$

where the collimating and the imaging-lens focal lengths are $f_{c}, f_{i}$, respectively, and the incident angles of the signal and reference beams $\theta_{S}, \theta_{R}$ are determined by the holographic imaging system. We also assume that $\Delta x_{p}, \Delta z_{d}^{\prime} \ll f_{c}, f_{i}$.

Figure 18 shows the theoretical angle selectivity of the diffraction intensity curve in Eq. (49), which is consistent with the numerical simulation intensity as the probing beam shifts along the $\hat{\mathbf{x}}$ direction. The numerical simulation of the 2-D diffracted patterns 
in Fig. 19 shows a linear spatial transformation of the probing point source, when the intensity is modulated because of angle selectivity.

\section{Selectivity in the Depth $\hat{\mathbf{z}}_{p}$ Direction}

As the probing point source shifts along the axis of the collimating lens in Fig. 16, the probing wave front for the hologram is no longer a plane wave. Instead it becomes a spherical wave with curvature determined by the collimating lens $f_{c}$ and the shifting $\Delta z_{p}$. The diffraction characteristics are determined by different spatial components of the probing beam, which has no analytic solution as do the cases in Section 3.

To get a simple analytic approximate formula for the intensity depth selectivity, we consider the diffracted intensity to be composed of the diffracted intensity from various spatial-frequency components in the incident probing beam. Ignoring the diffractedintensity changes along the degenerate $\hat{\mathbf{y}}_{p}$ direction and considering only the intensity diffraction efficiency for spatial-frequency component $\delta k_{x}$ governed by Eq. (49), we have the approximation

$$
\begin{aligned}
\eta\left(\Delta z_{p}\right) & \approx \frac{1}{\alpha} \int_{0}^{\alpha} \operatorname{sinc}^{2}\left(\frac{t}{\Delta \alpha}\right) \mathrm{d} t, \\
\alpha & =\frac{L \Delta z_{p}}{2 f_{c}^{2}} .
\end{aligned}
$$

The integral in Eq. (51) has a universal line shape as shown in Fig. 20 in which the function drops to a half-value at $\alpha=0.90 \Delta \alpha$. If the half-intensity shift is defined as the depth selectivity $\Delta z_{p 1 / 2}$, then

$$
\Delta z_{p 1 / 2}=1.80 \frac{f_{c}^{2}}{L} \Delta \alpha
$$

which gives $\Delta z_{p 1 / 2}=26 \lambda$ in the simulation conditions. Figure 20 shows the approximate intensity selectivity and the numerical simulation on the intensity integral over the image patterns, which are consistent with one another. Figure 21 shows the 2-D intensity diffraction pattern on the image plane as the point source at the original recording wavelength is shifted along the depth $\hat{\mathbf{z}}_{p}$ direction. The image pattern diffuses very fast in the $\hat{\mathbf{z}}^{\prime}, \hat{\mathbf{y}}^{\prime}$ directions while the intensity drops.

Figure 22 shows one experimental measurement of the transverse $\hat{\mathbf{x}}_{p}$ and depth $\hat{\mathbf{z}}_{p}$ intensity selectivity curves with a DuPont photopolymer $100-\mu \mathrm{m}$ thickness. Theoretical calculation of the corresponding selectivity in Eqs. (47) and (53), with the experimental parameters and refractive index $n=1.5$ for the polymer, matches the measurement very well. The higher sidelobe of the experimental data in the $\hat{\mathbf{x}}$ selectivity is due to saturation of the hologram (with $\eta>20 \%$ ). The depth-sectioning resolution $\left(2 \Delta z_{p 1 / 2}\right)$ is more than $800 \mu \mathrm{m}$ for the thin DuPont polymer. The smaller optical section depth can be achieved by thicker material and an objective lens with a larger numerical aperture.
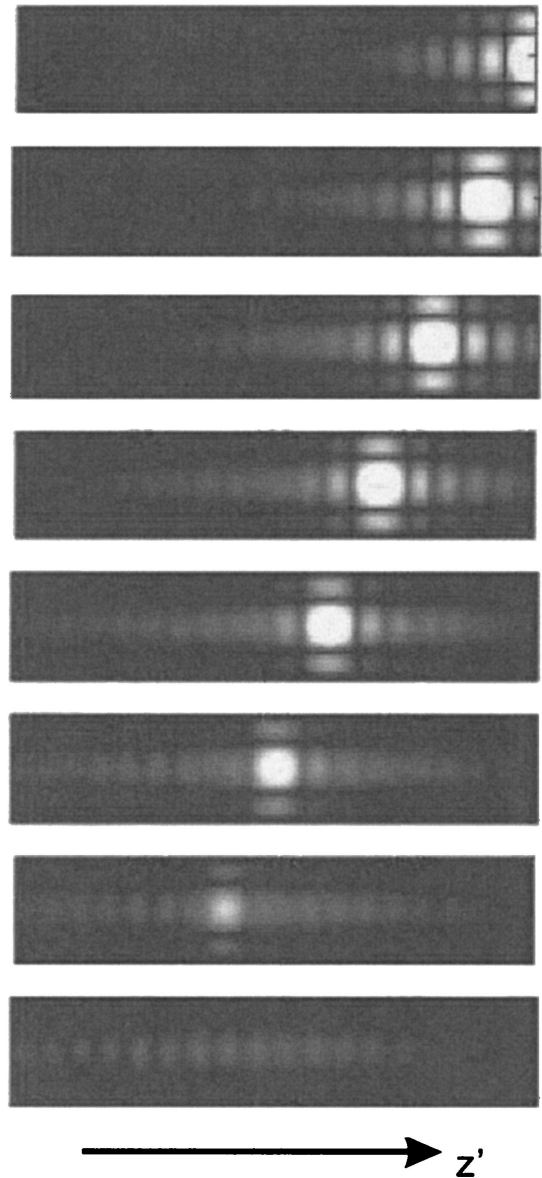

Fig. 19. Simulated intensity distribution patterns on the image plane of a general transmission geometry hologram in Fig. 16. The simulation conditions are the same as in Fig. 18. Top to bottom, the probing point source at the recording wavelength moves from the recording position to the $\hat{\mathbf{x}}_{p}$ direction by $2 \lambda /$ step. The image pattern intensity and the location change are as in Eqs. (49) and (47), respectively.

\section{Selectivity in Wavelength}

With a process similar to the angular selectivity in a transmission hologram as shown in Fig. 17, we get the response to a probing point source $\delta\left(\mathbf{r}_{p}=\mathbf{r}_{r}, \lambda_{p}=\right.$ $\left.\lambda_{r}+\Delta \lambda\right)$ on the image plane as a point image $\eta \delta\left(\mathbf{r}_{d}{ }^{\prime}\right.$ $\left.-\mathbf{r}_{r}{ }^{\prime}-\Delta z_{d}{ }^{\prime} \hat{\mathbf{z}}^{\prime}, \lambda_{p}\right)$ :

$$
\begin{aligned}
\frac{\Delta z_{d}^{\prime}}{f_{i}} & =-n \frac{\Delta \lambda}{\lambda} \frac{\sin \theta_{R n}+\sin \theta_{S n}}{\cos \theta_{R}}, \\
\eta\left(\frac{\Delta \lambda}{\lambda}\right) & =\operatorname{sinc}^{2}\left(\frac{\Delta \lambda}{\lambda \Delta \beta}\right), \\
\Delta \beta & =\frac{\lambda}{n D} \frac{\cos \theta_{R n}}{1-\cos \left(\theta_{S n}+\theta_{R n}\right)},
\end{aligned}
$$

where Eq. (56) is the well-known wavelength selectivity.

Figure 23 shows the intensity selectivity curve from Eq. (55) and is consistent with the simulation intensity integral over the 2-D distribution on the 


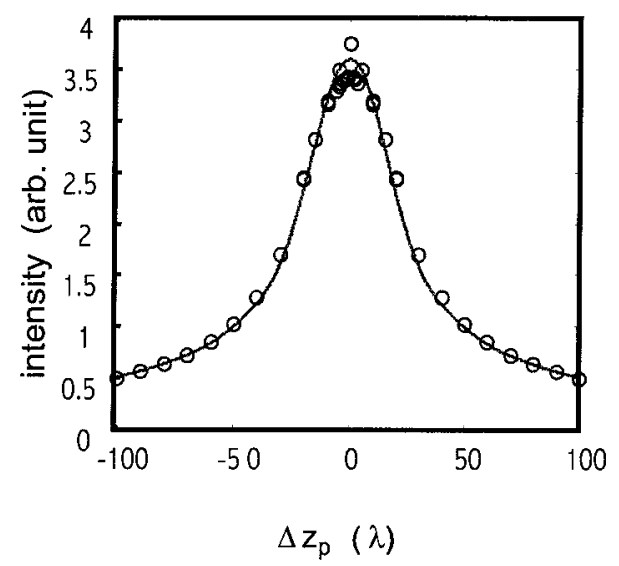

Fig. 20. Simulated diffraction intensity on the image plane of the general transmission geometry hologram in Fig. 16 as the probing point source shifts along the depth $\hat{\mathbf{z}}_{p}$ direction. The simulation conditions are the same as in Fig. 18: $\bigcirc$, numerical simulation; solid curve, approximate integral as in Eq. (51).

image plane. Figure 24 shows the 2-D image pattern as the probing wavelength is changed. The image pattern remains a point image while shifting along the $\hat{\mathbf{z}}_{d}{ }^{\prime}$ direction and decreases in intensity according to Eqs. (54) and (55).

If a chromatic point source is used as a probe at the recording position, the diffracted image pattern generated on the image plane will have sidelobes along the $\hat{\mathbf{z}}_{d}{ }^{\prime}$ direction with different wavelength components corresponding to Eq. (54) and Figs. 23 and 24. Figure 25 shows an experimental diffraction image when the hologram in Fig. 16 is probed with a whitelight point source. The intensity profile along the image plane $\hat{\mathbf{z}}_{d}{ }^{\prime}$ direction determines the image resolution of a chromatic point source in the objective space. Compared with the image resolution due to the object spatial selectivity along the $\hat{\mathbf{x}}_{p}$ direction as shown in Fig. 18, the image resolution of the chromatic effect is approximately $\times 2$ of the image reso-

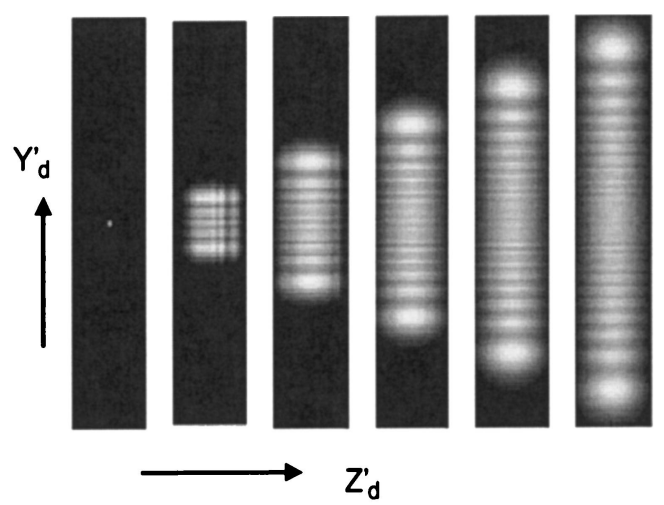

Fig. 21. Simulated diffraction intensity on the image plane of a general transmission geometry hologram in Fig. 16 as the probing point source shifts along the depth $\hat{\mathbf{z}}_{p}$ direction by $20 \lambda$ for each step, at the recording wavelength. The simulation conditions are the same as in Fig. 18.

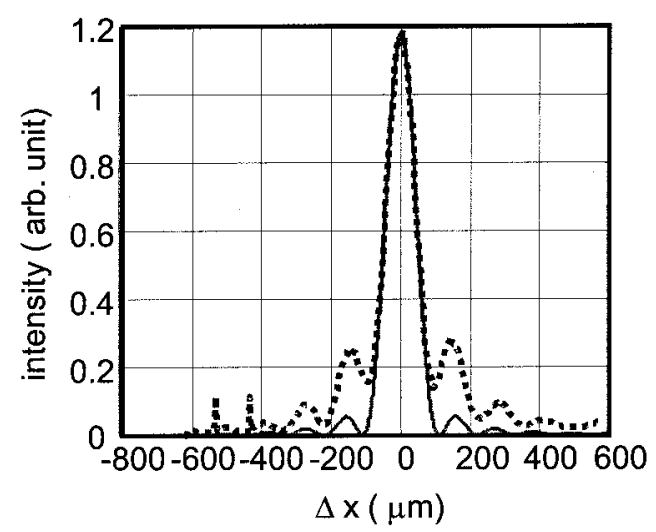

(a)

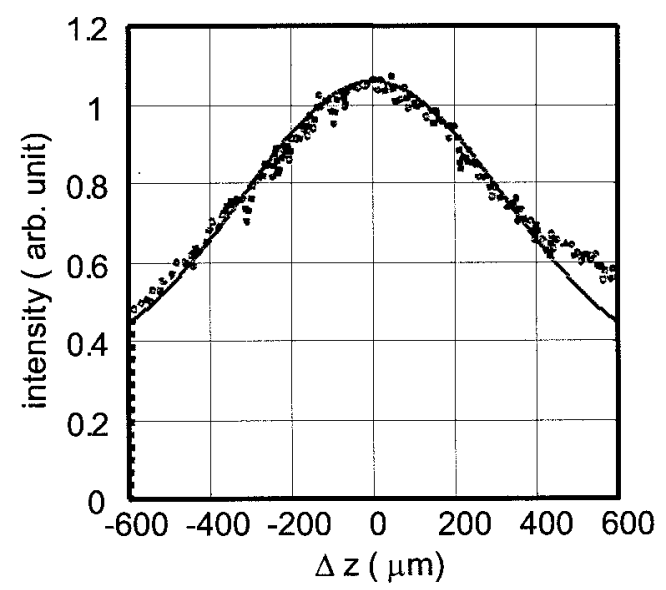

(b)

Fig. 22. Experimental measurements and theoretical intensity selective calculation of the shift selectivity of DuPont polymer 100 $\mu \mathrm{m}$ thick in the transmission geometry in Fig. 16. The collimating objective lens is $\times 10, N A=0.25$; the DuPont polymer is assumed to be $n=1.5$; and the wavelength is $488 \mathrm{~nm}$. (a) Shift selectivity in the $\hat{\mathbf{x}}_{p}$ direction, where Eq. (47) gives $\Delta x_{p}=104 \mu \mathrm{m}$ at the first null. (b) Depth selectivity in the $\hat{\mathbf{z}}_{p}$ direction; Eq. (53) gives $\Delta z_{p 1 / 2}=400 \mu \mathrm{m}$ at the half-magnitude.

lution due to the angle selectivity along $\hat{\mathbf{x}}_{p}$, which is determined by Eqs. (47)-(56):

$$
\begin{aligned}
& \frac{\Delta z_{d}^{\prime}\left(\frac{\Delta \lambda}{\lambda}=\Delta \beta\right)}{\Delta z_{d}^{\prime}\left(\frac{\Delta x_{p}}{f_{c}}=\Delta \alpha\right)} \\
& \quad=\frac{\left(\sin \theta_{R n}+\sin \theta_{S n}\right)\left(\tan \theta_{R n}+\tan \theta_{S n}\right) \cos \theta_{R n}}{1-\cos \left(\theta_{S n}+\theta_{R n}\right)},
\end{aligned}
$$

which has a value of 2 in the simulation conditions in Fig. 18.

\section{Selectivity in the Depth $\hat{\mathbf{z}}_{p}$ Direction from a Chromatic Source}

For observations with a chromatic source the image resolution along $\hat{\mathbf{z}}^{\prime}$ is dominated by wavelength selectivity as mentioned above. Similarly, for the 


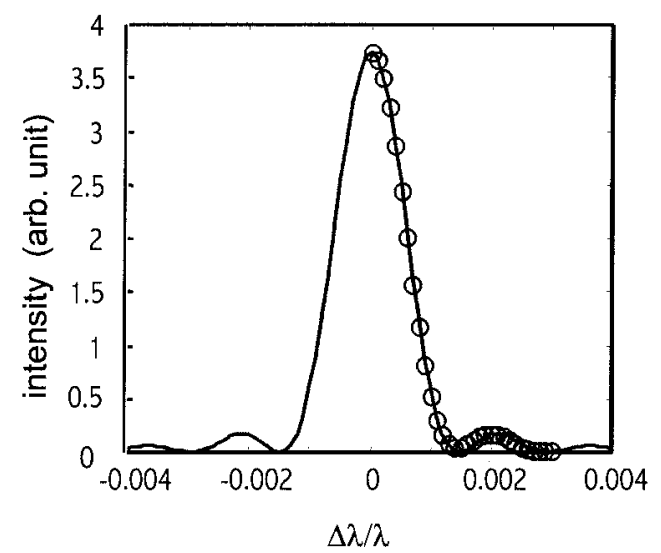

Fig. 23. Simulated diffraction intensity on the image plane of a general transmission geometry hologram in Fig. 16 as the wavelength of the probing point source changes at the original recording location. The simulation conditions are the same as in Fig. 18.

depth selectivity that determines the depth resolution of optical sectioning, the coupling between the depth selectivity at different wavelengths from the recording wavelength needs to be addressed. When the probing point source shifts along the $\hat{\mathbf{z}}_{p}$ direction at different wavelengths from the recording one, the intensity selectivity is simulated and compared with
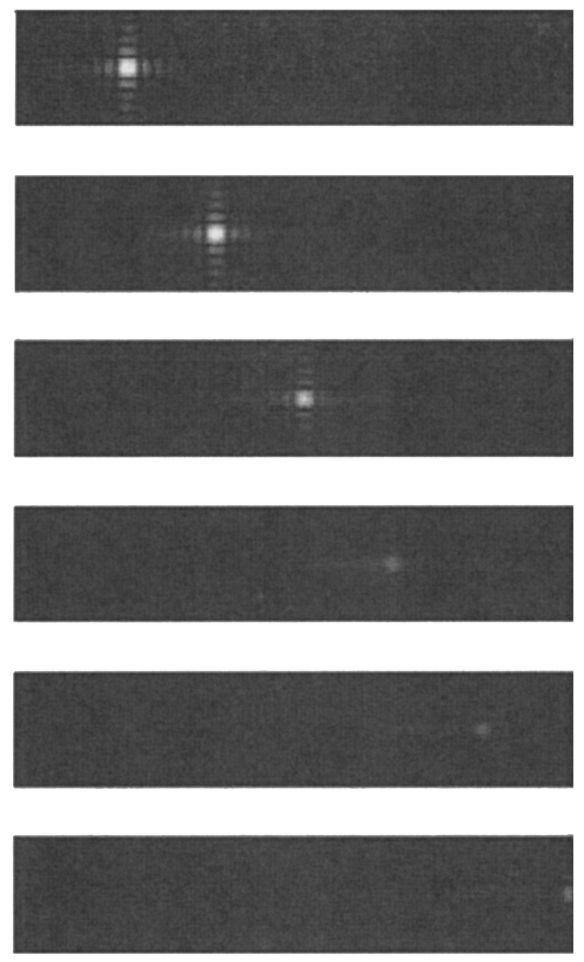

Fig. 24. Simulated intensity distribution patterns on the image plane of the general transmission geometry hologram in Fig. 16 as the probing point source changes wavelength from the recording wavelength by $\Delta \lambda / \lambda=-4 \times 10^{-4}$ for each step. The simulation conditions are the same as in Fig. 18.

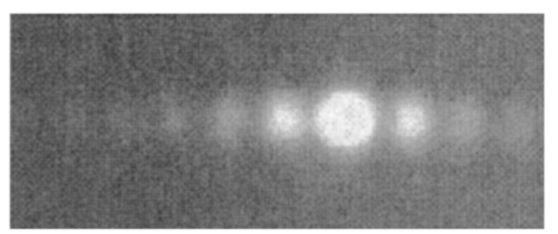

Fig. 25. Experimental measurement of the intensity distribution pattern on the image plane of a general transmission geometry hologram in Fig. 16 for a chromatic point source located at the original recording position. The full width of the first null of the intensity profile determines the spatial-image resolution along $\hat{\mathbf{z}}_{d}{ }^{\prime}$ on the image plane.

the depth selectivity at the recording wavelength in Fig. 26.

The intensity shift functions in the $\hat{\mathbf{z}}_{p}$ direction at different wavelengths are all within the sidelobe of the selectivity of the original recording wavelength. The depth resolution is dominated by the spatial selectivity given in Eq. (53).

\section{Selectivity along the $\hat{\mathbf{y}}_{p}$ Direction}

When the probing point source is shifted along $\hat{\mathbf{y}}_{p}$ as $\delta\left(\mathbf{r}_{p}=\mathbf{r}_{r}+\Delta \mathbf{y}_{p}, \lambda_{p}=\lambda_{r}\right)$, the response on the image plane is a point image $\eta \delta\left(\mathbf{r}_{d}{ }^{\prime}-\mathbf{r}_{r}{ }^{\prime}-\Delta \mathbf{r}_{d}{ }^{\prime}, \lambda_{p}\right)$ :

$$
\begin{aligned}
\eta & =\operatorname{sinc}^{2}\left(\frac{y_{p}{ }^{2}}{f_{c}^{2}} \frac{1}{\Delta \alpha_{y}}\right), \\
\Delta \mathbf{r}_{d}{ }^{\prime} & =-\frac{f_{i}}{f_{c}} \Delta y_{p} \hat{\mathbf{y}}^{\prime}-\frac{\Delta y_{p}{ }^{2}}{2 f_{c}{ }^{2}} f_{i} \hat{\mathbf{z}}_{d}{ }^{\prime}, \\
\Delta \alpha_{y} & =2\left(n^{2}-\frac{1}{4}\right)^{1 / 2} \frac{\lambda}{D},
\end{aligned}
$$

in the simulation geometry. Figure 27 shows the intensity selectivity curve, which is consistent with numerical simulation results. Figure 28 shows the

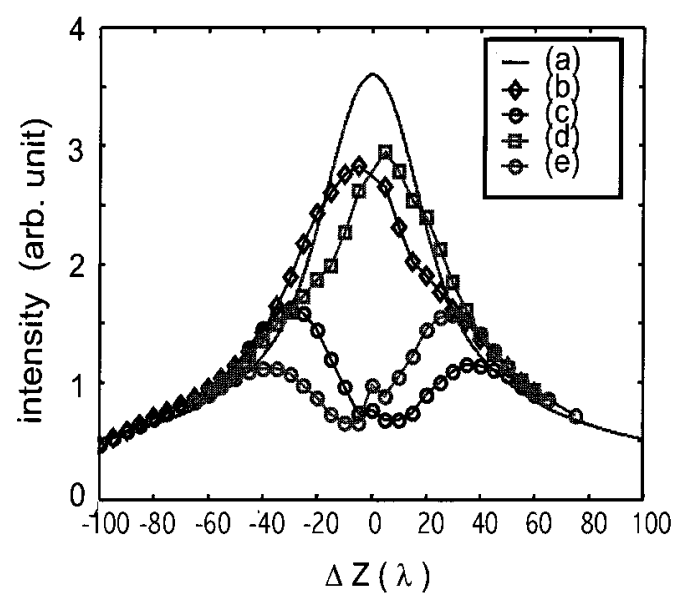

Fig. 26. Simulated diffraction intensity on the image plane of a general transmission geometry hologram in Fig. 16 as the probing point source shifts along the depth $\hat{\mathbf{z}}_{p}$ at different wavelengths: (a) original wavelength $(\Delta \lambda) / \lambda=0$; (b) $(\Delta \lambda) / \lambda=-5 \times 10^{-4}$; (c) $(\Delta \lambda) / \lambda=-10^{-3} ;(\mathrm{d})(\Delta \lambda) / \lambda=5 \times 10^{-4} ;(\mathrm{e})(\Delta \lambda) / \lambda=10^{-3}$. The simulation conditions are the same as in Fig. 18. 


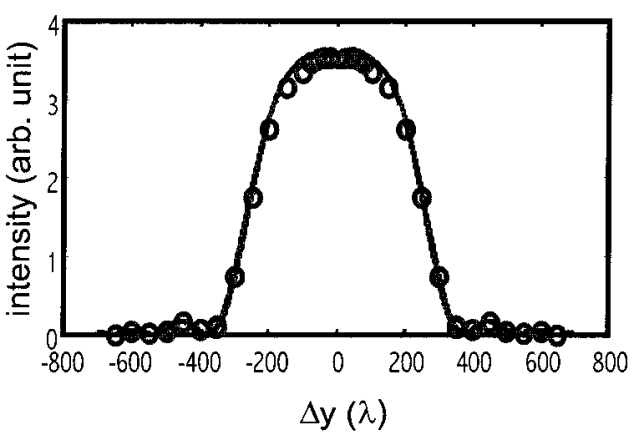

Fig. 27. Simulated diffraction intensity on the image plane of a general transmission geometry hologram in Fig. 16 as the probing point source shifts along the $\mathbf{y}_{p}$ direction at the original wavelength. The simulation conditions are the same as in Fig. 18.

2-D image pattern for point source shifting along the $\hat{\mathbf{y}}_{p}$ direction. The image pattern not only linearly shifts along the $\hat{\mathbf{y}}_{d}{ }^{\prime}$ direction but also drifts in the $-\hat{\mathbf{z}}_{d}{ }^{\prime}$ direction as determined by Eq. (59).

B. Degenerate Diffraction and Two-Dimensional Imaging Demonstration

In the simple transmission geometry with plane waves as the signal and reference, there are two dimensions where exact Bragg phase matching can be achieved. As the wave-vector $k$ space in Fig. 29 shows, for a single grating $K$ recorded by beams $\mathbf{R}_{1}$, $\mathbf{S}_{1}$, pairs of $\mathbf{R}_{2}, \mathbf{S}_{2}$ can also be Bragg phase matched with uniform diffraction efficiency with the same wavelength $k_{0}$ as in a recording. In addition, on a different $k$ sphere with a different wavelength $k_{1}$ in Fig. 29, there is a similar pair of $\mathbf{R}_{3}, \mathbf{S}_{3}$ with different
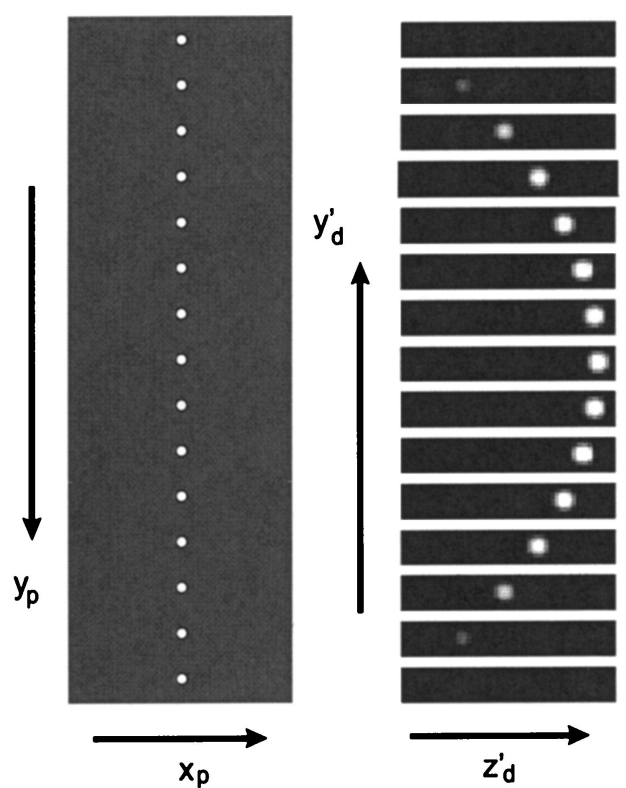

Fig. 28. Simulated diffraction image patterns on the Fourier plane of a general transmission geometry hologram in Fig. 16 as the probing point source shifts along the $\hat{\mathbf{y}}_{p}$ direction by $50 \lambda$ for each step at the original wavelength. The simulation conditions are the same as in Fig. 18.

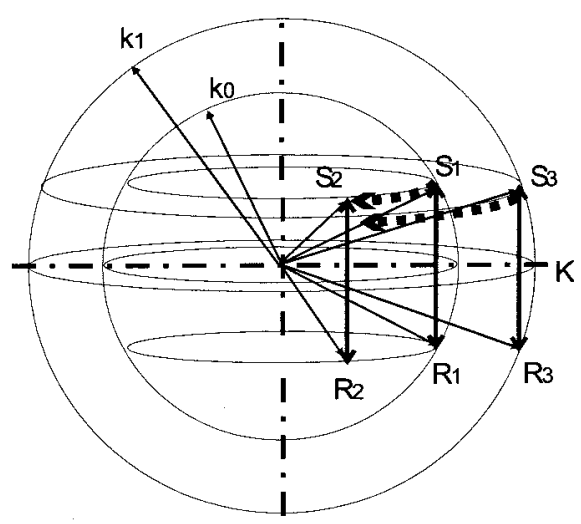

Fig. 29. Wave-vector $k$ sphere for a holographic grating $\mathbf{K}$ recorded by $\mathbf{R}_{1}, \mathbf{S}_{1}$ at wavelength $k_{0}$. The hologram can be Bragg phase matched by a pair of beams $\mathbf{R}_{2}, \mathbf{S}_{2}$ rotated around the $\mathbf{K}$ direction at the same wavelength. At a different wavelength $k_{1}$, there is another pair of beams $\mathbf{R}_{3}, \mathbf{S}_{3}$ with a corresponding tilting angle that can be Bragg phase matched while rotated around the $\mathbf{K}$ direction.

angles, which can be Bragg phase matched by the same holographic grating.

These two degeneracies lead to the linear response of the probing point source in Fig. 16, $\delta\left(\Delta \mathbf{r}_{p}, \Delta \lambda_{p}\right)$, onto the image plane $\delta\left(\Delta \mathbf{r}_{d}{ }^{\prime}, \Delta \lambda\right)$, when

$$
\begin{aligned}
\Delta \lambda_{p} & =0, \\
\Delta \mathbf{r}_{p} & =\Delta \mathbf{x}_{p}+\Delta \mathbf{y}_{p}, \\
\Delta \mathbf{r}_{d}{ }^{\prime} & =\Delta \mathbf{z}_{d}{ }^{\prime}+\Delta \mathbf{y}_{d}{ }^{\prime}, \\
\frac{\Delta x_{p}}{f_{c}} & =-\frac{\Delta z_{d}{ }^{\prime}}{f_{i}} \ll 1, \\
& =\frac{-\sin \theta+\left[\cos \theta^{2}-\left(n^{2}-\sin \theta^{2}\right) t^{2}\right]^{1 / 2}}{\sin \theta+\left[\cos \theta^{2}-\left(n^{2}-\sin \theta^{2}\right) t^{2}\right]^{1 / 2}}, \\
\frac{\Delta y_{p}}{f_{c}} & =-\frac{\Delta y_{d}{ }^{\prime}}{f_{i}}, \\
& =\frac{-(2)^{1 / 2}\left(n^{2}-\sin \theta^{2}\right)^{1 / 2} t}{\sin \theta+\left[\cos \theta^{2}-\left(n^{2}-\sin \theta^{2}\right) t^{2}\right]^{1 / 2}}
\end{aligned}
$$

or

$$
\begin{aligned}
\frac{\Delta x_{p}}{f_{c}} & =-\frac{\Delta \lambda_{p}}{\lambda}, \\
& =\frac{\Delta z_{d}{ }^{\prime}}{f_{i}},
\end{aligned}
$$

where the incident angle $\theta_{R}=\theta_{S}=\theta$ and the parameter $t$ can be any number with $(-1,1)$.

Assuming a 2-D object on the objective plane with a single-wavelength component, the optical sectioning by a single transmission hologram is a vertical curve given by Eqs. (65)-(67) on the objective plane, which is linearly transformed onto the image plane. For the same object with polychromatic illumination 


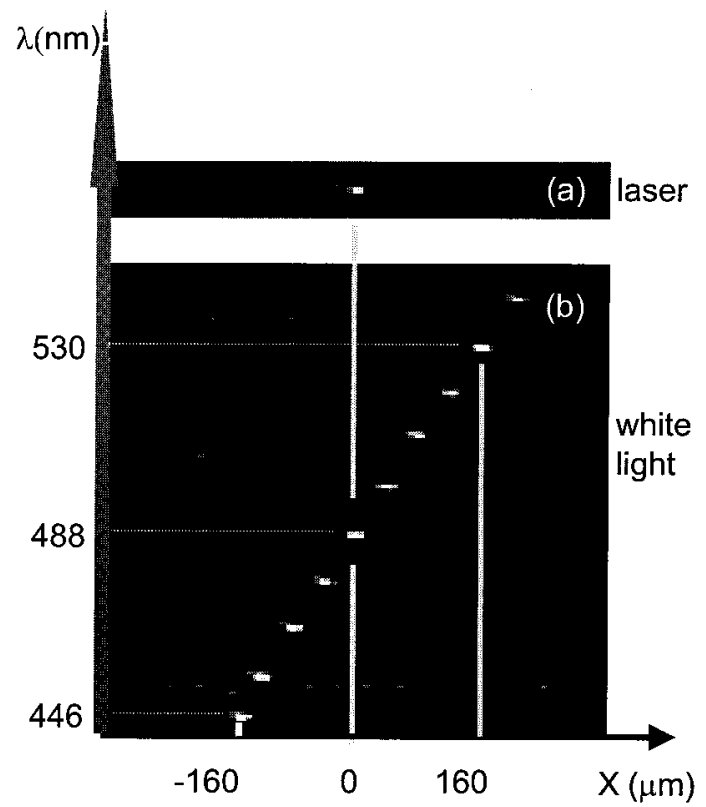

Fig. 30. Experimental demonstration of Bragg phase matching by wavelength-shift coupling in a transmission geometry in Fig. 16. The experimental parameters are as follows: $\mathrm{LiNbO}_{3}$ crystal thickness, $5 \mathrm{~mm}$; collimating objective lens, $\times 10, N A=0.25$; and recording wavelength, $488 \mathrm{~nm}$. (a) Image pattern while the probing pinhole is illuminated by $488 \mathrm{~nm}$. (b) Image pattern as the white-light-illuminated pinhole shifts in the $\hat{\mathbf{x}}_{p}$ direction. The image point shifts correspondingly in the $\hat{\mathbf{z}}_{d}{ }^{\prime}$ direction with a different wavelength component determined by Eqs. (68) and (69).

a 2-D hyperslice is optically sectioned from the 3-D information hyperspace (2-D spatial on the $\mathbf{x}_{p}-\mathbf{y}_{p}$ plane plus spectrum dimension) with the wavelength and $\mathbf{x}_{p}$ coupling relation in Eq. (68). This optical section is projected onto the image plane and gives us a 2-D image of the $\mathbf{x}_{p}-\mathbf{y}_{p}$ plane with a $\mathbf{z}_{d}{ }^{\prime}$ related color-filtering effect. The image spatial resolution is determined by Eqs. (47), (54), and (57) along the $\hat{\mathbf{x}}_{p}$ or $\hat{\mathbf{z}}_{d}{ }^{\prime}$ direction on the image plane. The opticalsectioning resolution is given by Eq. (53) for depth selectivity and Eq. (56) for wavelength selectivity.

Figure 30 demonstrates the degenerate Bragg phase-matching in Eqs. (68) and (69), where the image pattern of a probing point source shifting along the $\hat{\mathbf{x}}_{p}$ direction is recorded. When the probing point source is a $5-\mu \mathrm{m}$ pinhole illuminated with the same recording wavelength, the image pattern is a point pixel at the original reference focal point only when the pinhole is located at the recording location, as shown in Fig. 30(a). When the pinhole is illuminated by a white-light source and is shifting along the $\hat{\mathbf{z}}_{d}{ }^{\prime}$ direction, the image pattern is a pixel point with the wavelength determined by Eqs. (68) and (69), as shown in Fig. 30(b).

Figure 31 demonstrates the ability to form a 2-D image by a single-transmission hologram with degeneracies in spatial and wavelength dimensions. A 2 -D object mask is used as the probing source and is illuminated by the recording wavelength. Because of the degenerate direction along $\mathbf{y}_{p}$, a vertical line (a)

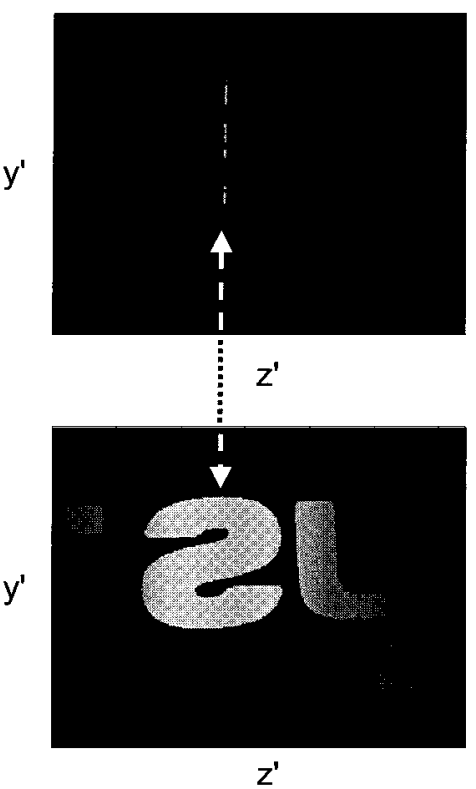

Fig. 31. Experimental demonstration of 2-D imaging by Bragg phase matching in the spatial and the wavelength-shift coupling dimension of a single transmission geometry hologram in Fig. 16. The experimental parameters are the same as in Fig. 30. (a) Image pattern of a 2-D mask across the original recording pointsource location and illuminated by $488 \mathrm{~nm}$. A vertical-line image is formed from spatial degeneracy along the $\hat{\mathbf{y}}_{p}$ direction. (b) Image pattern as the white light illuminates the 2-D mask. At a different $\hat{\mathbf{z}}_{d}{ }^{\prime}$ position, a different wavelength component Bragg phase matches and forms the 2-D color-coded image, determined by Eqs. (68) and (69).

image across the recording point is projected onto the image plane in Fig. 31(a), as discussed in Eqs. (63)(67). However, for a chromatic object (the mask illuminated by a white-light source) the additional degeneracy by different wavelength components forms the 2-D color-coded image in Fig. 31(b), where the wavelength- $\mathbf{z}_{d}{ }^{\prime}$ relation is shown in Fig. 30(b).

\section{Experimental Three-Dimensional Imaging and Discussion}

\section{A. Three-Dimensional Imaging}

Holographic imaging makes use of the spatial and wavelength degenerate Bragg-phase-matching effects in a single hologram for optical sectioning a 2-D slice from a 4-D objective hyperspace (3-D spatial plus spectral dimensions) and projecting it linearly onto a 2-D detector, where each pixel corresponds to a texel volume in the object 4-D hyperspace. Compared with a traditional imaging system that sections a 2-D slice from three spatial dimensions and projects a single voxel volume onto an individual pixel onto the detector, the holographic imaging extracts additional information on the spectra at the expense of a lower photon flow for each pixel corresponding to a texel volume.

The other major advantage of holographic imaging is that the volume hologram selectively diffracts only the Bragg-matched signal components and has little 

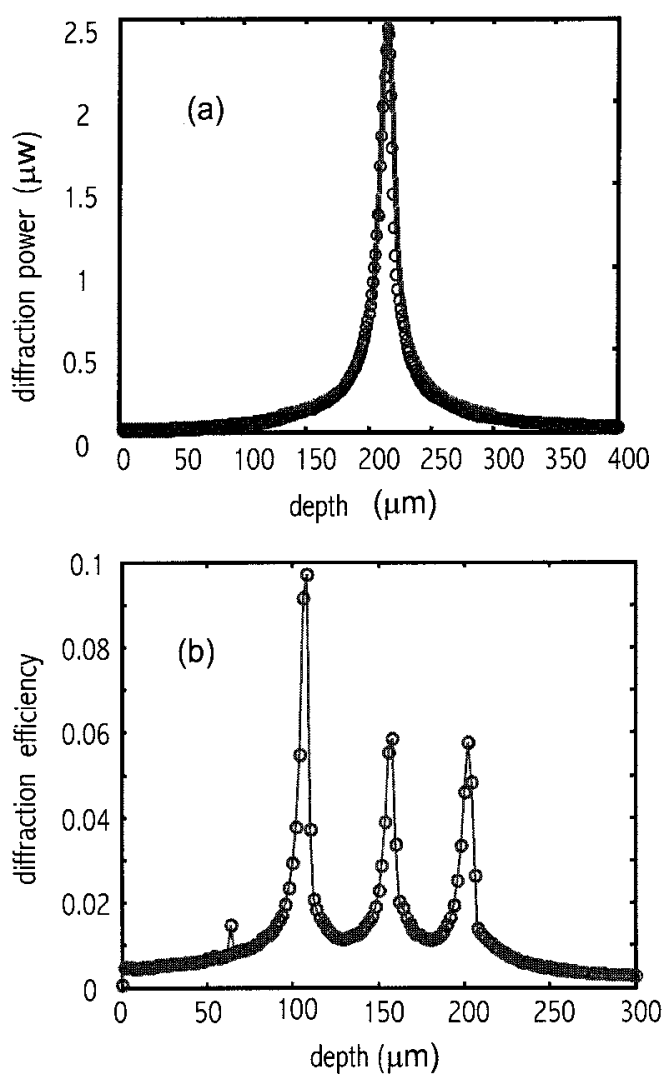

Fig. 32. Depth-selectivity measurement and comparison with the theoretical prediction in a $\mathrm{PQ}$ polymer $2 \mathrm{~mm}$ thick. The experimental setup is as in Fig. 16: collimating objective lens, $\times 40 ; N A$ $=0.65$; wavelength, $488 \mathrm{~nm}$. (a) Single strong hologram with a diffraction efficiency of $>15 \%$. The depth selectivity is consistent with the theoretical calculation by Eq. (51). (b) Three holograms multiplexed with a different recording depth $z_{r} 50 \mu \mathrm{m}$ apart.

effect on other components. With holographic multiplexing techniques, multiple holograms can be built into the system simultaneously and can process different signal components at the same time. When different slices are sectioned from the 4-D hyperspace, the number of texels detected at one time are much larger than the number of voxels a normal 2-D imaging system can achieve. This increases the information-collection rate, however, with the requirement of a larger photon flux for a holographic imaging system to have the same signal-to-noise ratio as each pixel on the 2-D image sensor.

Here we demonstrate multiple-slice sectioning at once by multiplexing holograms, which achieves the ability to extract 3-D spatial information at a single shot. The holograms are recorded on a 2-mm-thick phenanthrenequinone polymer, which gives a depth selectivity of $\Delta z_{p 1 / 2}=2 \mu \mathrm{m}$ according to Eq. (53) for a 488-nm wavelength and an objective lens $\times 40, N A=$ 0.65 .

Figure 32(a) shows the depth selectivity of a single strong hologram in the transmission geometry with a diffraction efficiency of $>15 \%$. The depth selectivity is consistent with the theoretical calculation in Eq. (51) in the experimental condition. Figure 32(b)

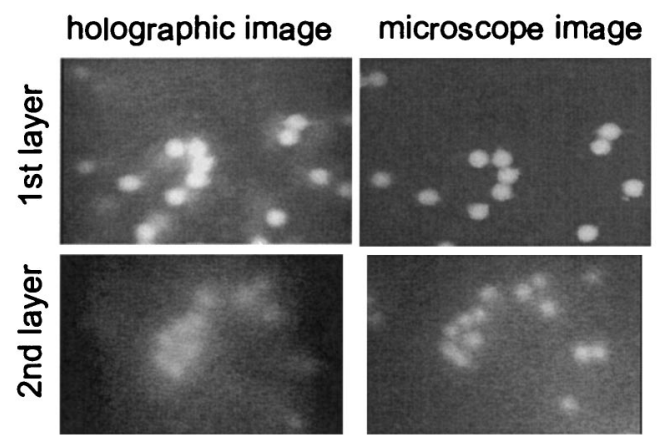

Fig. 33. Images of fluorescent microspheres (15 $\mu \mathrm{m}$ in diameter $)$ excited by $488 \mathrm{~nm}$ and emitting at a peak fluorescent wavelength of $515 \mathrm{~nm}$, by holographic and normal microscope imaging systems with the same collimating and imaging lenses.

shows the depth selectivity of three multiplexed holograms in the same $\mathrm{PQ}$ polymer with an average diffraction efficiency of $7 \%$. The three holograms are recorded with a different recording depth $z_{r}$ at a $50-\mu \mathrm{m}$ distance. Also the reference beams have different angles for the three holograms, which optically section three slices at different depths from the 3-D objective spatial dimension and project them onto a different area of the image sensor. The three peaks in Fig. 32(b) have good separation, but the broad and slowly-decreasing sidelobe of the depth selectivity causes background noise in the images.

To demonstrate the ability of the 3-D imaging of real 3-D objects, we use fluorescent microspheres as our observation targets. Fluorescent microspheres are polyspheres doped with certain dyes that can be excited by some wavelengths and emit fluorescent light. They are widely used in biomedical research in confocal microscopes and fluorescent microscopes. ${ }^{26,54,55}$

We use $15-\mu \mathrm{m}$-diameter microspheres in our experiments, excited by $488 \mathrm{~nm}$ and emitting fluorescent light at a central peak wavelength of $515 \mathrm{~nm} .{ }^{58}$ Figure 33 shows a comparison of the imaging quality between holographic imaging and normal microscope imaging. Two layers of microspheres are sealed in solid polymer with a distance of $100 \mu \mathrm{m}$ between them. The visual image quality of single-hologram imaging and a normal microscope system with the same collimating and imaging lenses is comparable, owing to the high spatial selectivity achieved by the holographic imaging elements. However, because of the intrinsic color-filtering effect in holographic imaging, the signal level for the detector pixels in holographic imaging is much lower than the normal microscope.

To demonstrate the multiple optical sectioning and the real-time imaging of 3-D space, a liquid sample of fluorescent microspheres is used. Figure 34(a) shows the optical sectioning and imaging by a single hologram in the $\mathrm{PQ}$ polymer in Fig. 32(a). With three holograms multiplexed in the $\mathrm{PQ}$ polymer as in Fig. 34(b), three optical sectionings from different 


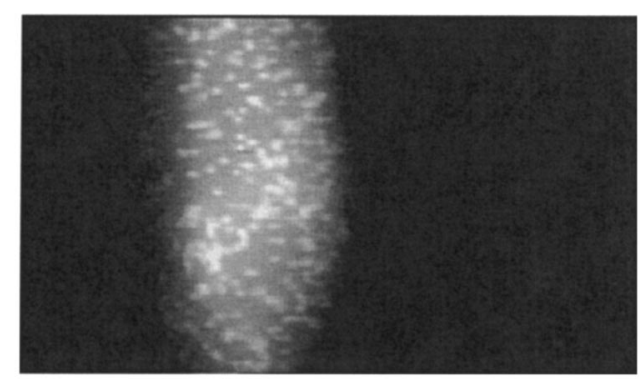

(a)

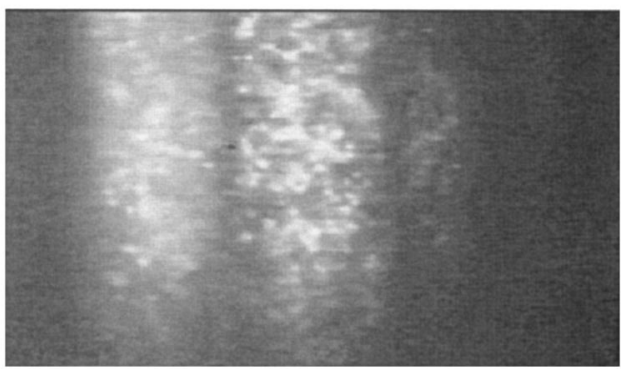

(b)

Fig. 34. Images of fluorescent microspheres (15 $\mu \mathrm{m}$ in diameter) in a liquid sample, excited by $488 \mathrm{~nm}$ and emitting at a peak fluorescent wavelength of $515 \mathrm{~nm}$ : (a) with a single hologram with the depth-sectioning ability as in Fig. 32(a); (b) with three multiplexed holograms for imaging three optical sections of different depth.

depths are projected onto the detector in real time at the same time in Fig. 34(b).

\section{B. Discussion}

To design a practical holographic imaging module, several important issues are to be addressed.

(1) Resolution: Owing to the existence of collimating and imaging lenses, the resolution of a holographic 3-D imaging system cannot achieve a higher resolution than the normal microscope. Also, the aberrations and the finite space-bandwidth product of these lenses limit the objective space volume from which the signals can be collected and projected onto the holograms.

New architectures and more sophisticated computer-designed holographic patterns could directly image the object without collimating lenses. Further investigation and theoretical and experimental verification are necessary to explore the potentials of such approaches.

(2) Photon efficiency: As we mentioned above, 4-D hyperspace sectioning causes the photon counts on each detector to decrease, which leads to a lower signal-to-noise ratio, or a higher photon flux requirement. This might be a problem for fluorescent samples because of the photobleaching effect on the fluorophores. This is especially a problem for wavelength sectioning, where volume holograms have typically a high wavelength selectivity and decrease the photon efficiency for spatial signal detection. Replacing the transmission geometry hologram in Fig. 16 with a reflection geometry hologram can provide a similar imaging system with even higher wavelength selectivity, which has also been demonstrated by experiments. For most of the applications in the microscopes the spectral information resolution required is far less than our current implementation of holographic imaging.

(3) Strong hologram multiplexing: To keep the photon efficiency as high as possible, we require the imaging holograms to have a large diffraction efficiency, which is not an easy task for most volume holographic materials. Photopolymers are a good choice only if the strong holograms in them can achieve a good wave-front reconstruction quality or a high spatial selectivity for the spatial opticalsectioning resolution. Localized recording in doubly doped photorefractive crystals ${ }^{57-61}$ is another potential method for overcoming this problem.

\section{Conclusion}

In summary, we have studied two holographic imaging architectures in terms of their spatial-spectral selectivities, 2-D diffracted image patterns, and degenerate Bragg imaging capabilities. We have developed a numerical model to simulate the diffraction-intensity distribution of any volume holographic structure. From the simulation and experiments we have verified the analytic approximation that gives simple theoretical equations describing the characteristics of these two holographic imaging architectures. With a collimating objective lens we have achieved the high-resolution holographic spectral imaging of 3-D objects with a transmission geometry hologram. The same architecture can be applied to reflection and 90-deg geometry holograms. Using multiplexed holograms, we have demonstrated the flexibility of imaging different spatial-spectral locations of a 3-D object simultaneously and projecting them onto single or multiple detectors.

W. Liu and D. Psaltis thank the National Science Foundation (NSF) Engineering Research Center of Neuromorphic Systems and the Defense Advanced Research Projects Agency (DARPA) for financial support for these efforts at Caltech. G. Barbastathis thanks NSF and the AFRL Munitions Directorate, Eglin AFB, for funding in part at the Massachusetts Institute of Technology.

\section{References}

1. D. Gabor, "Associative holographic memories," IBM J. Res. Dev. 13, 156-159 (1969).

2. E. N. Leith, A. Kozma, J. Upatnieks, J. Marks, and N. Massey, "Holographic data storage in three-dimensional media," Appl. Opt. 5, 1303-1311 (1966).

3. D. Psaltis, "Parallel optical memories," Byte 17, 179 (1992).

4. D. Psaltis and F. Mok, "Holographic memories," Sci. Am. 273, 70-76 (November 1995).

5. J.-J. P. Drolet, E. Chuang, G. Barbastathis, and D. Psaltis, "Compact, integrated dynamic holographic memory with refreshed holograms," Opt. Lett. 22, 552-554 (1997). 
6. G. W. Burr, H. Coufal, J. A. Hoffnagle, C. M. Jefferson, M. Jurich, B. Marcus, and R. M. Macfarlane, "Optical data storage enters a new dimension," Phys. World 13, 37-42 (July 2000).

7. D. Psaltis and G. W. Burr, "Holographic data storage," Computer 31(2), 52 (1998).

8. E. Chuang, W. H. Liu, J.-J. P. Drolet, and D. Psaltis, "Holographic random access memory," Proc. IEEE 87, 1931-1940 (1999).

9. D. Psaltis, "Coherent optical information systems," Science 298 (5597), 1359-1363 (2002).

10. D. Psaltis, D. Brady, X. G. Xu, and S. Lin, "Holography in artificial neural networks," Nature (London) 343, 325-330 (1990).

11. H.-Y. S. Li, Y. Qiao, and D. Psaltis, "Optical network for realtime face recognition,” Appl. Opt. 32, 5026-5035 (1993).

12. D. Psaltis and Y. Qiao, "Adaptive multilayer optical networks," Prog. Opt. 31, 227-261 (1993).

13. M. Levene, G. J. Steckman, and D. Psaltis, "Method for controlling the shift invariance of optical correlators," Appl. Opt. 38, 394-398 (1999).

14. A. Pu, R. Denkewalter, and D. Psaltis, "Real-time vehicle navigation using a holographic memory," Opt. Eng. 36, 2737-2746 (1997).

15. B. Javidi and E. Tajahuerce, "Three-dimensional object recognition by use of digital holography," Opt. Lett. 25, 610-612 (2000).

16. H. Lee, X.-G. Gu, and D. Psaltis, "Volume holographic interconnections with maximal capacity and minimal cross talk," J. Appl. Phys. 65, 2191-2194 (1989).

17. D. J. Brady and D. Psaltis, "Holographic interconnections in photorefractive waveguides," Appl. Opt. 30, 2324-2333 (1991).

18. K. L. Tan, W. A. Crossland, and R. J. Mears, "Dynamic holography for optical interconnections. I. Noise floor of lowcross-talk holographic switches," J. Opt. Soc. Am. A 18, 195204 (2001).

19. K. L. Tan, S. T. Warr, I. G. Manolis, T. D. Wilkinson, M. M. Redmond, W. A. Crossland, R. T. Mears, and B. Robertson, "Dynamic holography for optical interconnections. II. Routing holograms with predictable location and intensity of each diffraction order," J. Opt. Soc. Am. A 18, 205-215 (2001).

20. J. Upatnieks, A. V. Lugt, and E. N. Leith, "Correction of lens aberrations by means of holograms," Appl. Opt. 5, 589-593 (1966).

21. E. N. Leith and G. J. Swanson, "Holographic aberration compensation with partially coherent light," Opt. Lett. 7, 596-598 (1982).

22. M. R. Taghizadeh, P. Blair, B. Layet, I. M. Barton, A. J. Waddie, and N. Ross, "Design and fabrication of diffractive optical elements," Microelectron. Eng. 219-242 (1997).

23. A. Stadelmaier and J. H. Massig, "Compensation of lens aberrations in digital holography," Opt. Lett. 25, 1630-1632 (2000).

24. J. Michaells, C. Hettich, J. Mlynek, and V. Sandoghdar, "Optical microscopy using a single-molecule light source," Nature (London) 405, 325-327 (2000).

25. E. Betzig and R. J. Chichester, "Single molecules observed by near-field scanning optical microscopy," Science (London) 262, 1422-1425 (1993).

26. B. Herman and J. J. Lemasters, Optical Microscopy (Academic, San Diego, Calif., 1993).

27. A. Diaspro, S. Annunziata, M. Raimondo, P. Ramoino, and M. Robello, "A single-pinhole confocal laser scanning microscope for 3-d imaging of biostructures," IEEE Eng. Med. Biol. Mag. 18, 106-110 (1999).

28. T. Zhang and I. Yamaguchi, "Three-dimensional microscopy with phase-shifting digital holography," Opt. Lett. 23, 12211223 (1998).
29. N. V. Joshi and H. Medina, "Multiple beam interference confocal microscopy: tool for morphological investigation of a living spermatozoon," Microsc. Microanal. 6, 471-477 (2000).

30. G. Barbastathis and D. J. Brady, "Multidimensional tomographic imaging using volume holography," Proc. IEEE 87, 2098-2120 (1999).

31. W. H. Liu, D. Psaltis, and G. Barbastathis, "Real-time spectral imaging in three spatial dimensions," Opt. Lett. 27, 854-856 (2002).

32. M. Born and E. Wolf, Principles of Optics, 6th ed. (Cambridge University, Cambridge, England, 1980).

33. W. H. Liu and D. Psaltis, "Pixel size limit in holographic memories," Opt. Lett. 24, 1340-1342 (1999).

34. G. Barbastathis, M. Balberg, and D. J. Brady, "Confocal microscopy with a volume holographic filter," Opt. Lett. 24, 811813 (1999)

35. M. Levene, "Optics in neural computation" Ph.D. dissertation (California Institute of Technology, Pasadena, Calif., 1998).

36. J. W. Goodman, Introduction to Fourier Optics, 2nd ed. (McGraw-Hill, New York, 1996)

37. W. H. Liu, "Holographic resolution and its application in memory and imaging," Ph.D. dissertation (California Institute of Technology, Pasadena, Calif., 2001).

38. P. J. van Heerden, "A new optical method of storing and retrieving information," Appl. Opt. 2, 387-392 (1963).

39. F. T. S. Yu, S. Wu, A. W. Mayers, and S. Rajan, "Wavelength multiplexed reflection matched spatial filters using $\mathrm{LiNbO}_{3}$," Opt. Commun. 81, 343-347 (1991).

40. G. A. Rakuljic, V. Leyva, and A. Yariv, "Optical data storage by using orthogonal wavelength-multiplexed volume holograms," Opt. Lett. 17, 1471-1473 (1992).

41. J. Rosen, M. Segev, and A. Yariv, "Wavelength-multiplexed computer-generated volume holography," Opt. Lett. 18, 744746 (1993)

42. S. Yin, H. Zhou, F. Zhao, M. Wen, Z. Zhang, and F. T. S. Yu, "Wavelength-multiplexed holographic storage in a sensitive photorefractive crystal using a visible-light tunable diode laser," Opt. Commun. 101, 317-321 (1993).

43. K. Curtis, A. Pu, and D. Psaltis, "Method for holographic storage using peristropic multiplexing," Opt. Lett. 19, 993-994 (1994).

44. G. Barbastathis, M. Levene, and D. Psaltis, "Shift multiplexing with spherical reference waves," Appl. Opt. 35, 2403-2417 (1996).

45. D. Brady and D. Psaltis, "Control of volume holograms," J. Opt. Soc. Am. A 9, 1167-1182 (1992).

46. D. Psaltis, M. Levene, A. Pu, G. Barbastathis, and K. Curtis, "Holographic storage using shift multiplexing," Opt. Lett. 20, 782-784 (1995).

47. H.-Y. S. Li and D. Psaltis, "Three dimensional holographic disks," Appl. Opt. 33, 3764-3774 (1994).

48. G. Barbastathis and D. Psaltis, "Shift-multiplexed holographic memory using the two-lambda method," Opt. Lett. 21, 429 431 (1996).

49. H. Külich, "Reconstructing volume holograms without image field losses," Appl. Opt. 30, 2850-2857 (1991).

50. X. H. Zhang, J. J. Xu, Q. Sun, S. Liu, G. Q. Zhang, H. J. Qiao, F. F. Li, and G. Y. Zhang, "Dual-wavelength nonvolatile holographic storage," Opt. Commun. 180, 211-215 (2000).

51. E. Chuang and D. Psaltis, "Storage of 1000 holograms with use of a dual-wavelength method," Appl. Opt. 36, 8445-8454 (1997).

52. R. DeVre, J. F. Heanue, K. Gurkan, and L. Hesselink, "Transfer functions based on Bragg detuning effects for imagebearing holograms recorded in photorefractive crystals," J. Opt. Soc. Am. A 13, 1331-1344 (1996).

53. G. Barbastathis, "Intelligent holographic databases," Ph.D. 
dissertation (California Institute of Technology, Pasadena, Calif., 1998).

54. B. Matsumoto, Methods in Cell Biology (Academic, San Diego, Calif., 1993).

55. G. Isenberg, Modern Optics, Electronics, and High Precision Techniques in Cell Biology (Springer-Verlag, New York, 1998).

56. I. Molecular Probes, in Handbook of Fluorescent Probes and Research Chemicals, 7th ed., R. P. Haugland, ed. (http:www. probes.com, 2001).

57. C. Moser, "Optical information processing in crystals and polymers. Three dimensional wavefront sensors," Ph.D. dissertation (California Institute of Technology, Pasadena, Calif., 2001).
58. A. Adibi, "Persistent holographic storage in photorefractive crystals," Ph.D. dissertation (California Institute of Technology, Pasadena, Calif., 2000).

59. K. Buse, A. Adibi, and D. Psaltis, "Nonvolatile holographic storage in doubly doped lithium niobate crystals," Nature (London) 393, 665-668 (1998).

60. C. Moser, I. Maravic, B. Schupp, A. Adibi, and D. Psaltis, "Diffraction efficiency of localized holograms in doubly doped $\mathrm{LiNbO}_{3}$ crystals," Opt. Lett. 25, 1243-1245 (2000).

61. C. Moser, B. Schupp, and D. Psaltis, "Localized holographic recording in doubly doped lithium niobate," Opt. Lett. 25, 162$164(2000)$. 